\title{
Losing Face
}

\author{
Thomas Gall* and David Reinstein ${ }^{\dagger}$
}

June 25, 2017

\begin{abstract}
When Al makes an offer to Betty that Betty observes and rejects, Al suffers what we will call "loss-of-face", losing utility directly or through reputation. This can lead to inefficiency in bilateral matching problems, e.g., the marriage market, research partnering, and international negotiations. We offer a simple model with asymmetric information, continuous signals of binary types, and linear marriage payoffs. We compare a benchmark to a case where a male suffers a discrete loss if he plays accept and his partner plays reject. This threat makes rejecting strictly preferred to rejection, inefficiently reducing offers, making low types "reverse snobs", and making a "high types always reject" equilibrium stable. It makes males more selective than females and increases marriage assortativeness. The benchmark-no loss of face - can be restored through a "Conditionally Anonymous Environment" revealing only when both parties say "yes"; or by letting the side that is invulnerable to loss-of-face move second.
\end{abstract}

Keywords: Matching, marriage markets, anonymity, reputation, adverse selection, Bayesian games, emotions. JEL codes: D83, D03

\section{Introduction}

Matching markets are widespread, ranging from marriage, to employment, to business partnerships, to friendship-formation (Burdett and Coles, 1999). In such markets, the fear of rejection - in particular, the fear of having someone who rejects you know that you proposed to them - can lead to inefficiency. An individual may not ask someone out on a date, ask for a study partner, apply for a job, make a business proposition, propose a paper co-authorship, or suggest a peace treaty, because he does not want the other party to learn of his interest and turn him down. This may have a direct psychological cost, as in our main model. It may also have consequences for reputation and future play; as we show in the appendix, the former can represent a reduced form of the latter. In general, we call the disutility from this outcome "loss of face" (LoF).

Unlike standard problems of information asymmetry, LoF can be avoided, and outcomes potentially improved, by changing the information structure. For example, speed dating agencies often ask participants to mark the partners whom they are interested in, and then inform only

\footnotetext{
${ }^{*}$ Department of Economics, University of Southampton

${ }^{\dagger}$ corresponding author, Department of Economics, University of Exeter, d.reinstein@exeter.ac.uk
} 
those couples who both marked each other. This eliminates the risk of revealing one party's unrequited interest to the other. ${ }^{1}$

To understand the nature of the possible inefficiency, consider a game where each player knows her own type and gets an imperfect signal of her "partner's" type. Each can choose to "accept" or "reject" and if both accept, they are "married". Payoffs to marriage increase in the partner's type, while payoffs to remaining single increase in one's own type (this is specified precisely in our model). The game may be simultaneous or sequential, and the outcome (actions and payoffs) becomes common knowledge after all actions have been taken. Here, there may be a set of mutually-beneficial transactions that would occur without LoF, but do not occur with LoF because:

1. the proposer does not know for sure whether the other party will accept or reject and

2. a high enough probability of rejection can outweigh the expected gains to a successful transaction.

LoF depends on the (terminal) information structure, and it transforms material payoffs into psychological payoffs in a straightforward way. When a man accepts a woman and she rejects him, his material payoffs from this one-shot game are the same no matter what beliefs or information either party has. However, with LoF, his psychological payoffs are lower when she rejects him and she knows (for sure) that he accepted her. In other words, the other player's information about the history of play is a component of a player's utility function. ${ }^{2}$ For simplicity, we assume that LoF only occurs when the other player knows for certain you accepted and they rejected.

The effects of LoF are additional to the standard problems of asymmetric information (e.g., where a buyer refuses to purchase a perfectly good car because he expects it to be a "lemon"). In our model, players may reject potential partners even where their expected utility from a partnership is positive, for fear that if they played "Accept" the other party would reject them and they would lose face. Self-image also does not play a role in our model: a player's own inference does not depend on whether the other player learns of her offer. Thus, our "fear of losing face" is distinct from the motives discussed in Koszegi (2006) that may lead to over- or underconfident task choice. ${ }^{3}$

\footnotetext{
${ }^{1}$ An ad-hoc examination of eight UK-based speed-dating agencies (Speed Dater, Click Singles, Slow Dating, X Factor Dates, Ditch or Date?, When the Music Stops, Fast Love, and Date \& Dash) found that four reveal only mutual "yes,yes" matches (one of these also reveals the number of ticks you got), two reveal anyone who ticked you, one also reveals "warm; yes, maybe" matches, and one allows you to pay a fee to see who ticked you even though you didn't tick them (a "second chance"). Three of these also allow "friendship matches" of some form.

${ }^{2}$ Strictly speaking, this assumption puts our model into the category of a psychological game, as modeled by Battigalli and Dufwenberg (2009), in which my payoffs may depend on another player's beliefs about my action. However, in our model, for a given (exogenous) information structure, the relevant beliefs (the "terminal information sets", equivalent to the "terminal histories") are a one-to-one mapping from the players' actions; thus our analysis is standard. We assume that the structure of this terminal information is common knowledge. We refer to these as "terminal information sets" to avoid confusion with the standard terminology, in which information sets are only defined in connection with decision nodes. The simplicity of our game means we do not have to worry about, e.g., actions responding to equilibrium beliefs responding to actions.

${ }^{3}$ To state this a different way, our LoF is not because "Al's self-esteem suffers when he learn that Betty deems him to be low quality." The Conditionally Anonymous Environment, described below, can not shelter Al from learning this; if Al plays "Accept" he will always learn what Betty chose. Our LoF comes from Al's dislike-when Betty rejects him - of Betty knowing that $\mathrm{Al}$ saw her as a good partner and knowing that he made an offer to her. Future work could unpack this. Betty knowing that Al chose "accept" informs Betty about Al's quality, affecting his reputation, which he may value directly and/or which may impact my future payoffs (see C.7). It also may be undesirable because of reciprocity motives (Falk and Fischbacher, 2006): Al may want to repay
} 
If loss of face depends on whether the player who rejects knows whether the other player accepted her (i.e., it depends on the terminal information sets) then it can be avoided by changing the information structure so that players only learn about each other's behavior if they both play Accept. Now, after playing accept, $A l$ will still be able to infer if Betty rejected him, but Betty will not know that $\mathrm{Al}$ accepted her; knowing this, he will not suffer a loss of face. Thus, while Al's self-image can not be easily preserved, his face can be. We call such setups Conditionally Anonymous Environments or CAEs.

We mainly consider the fear of LoF as a primitive motive: this is particularly relevant to one-shot games where no outside parties observe the results. There is a special loss from the combined knowledge that you accepted somebody, but they rejected you. This may be a product of evolutionary factors, or perhaps an automatic internalization of a reputation motive. ${ }^{4}$ We present evidence below that a desire not to lose face is a primal human concern.

However, our key results carry over to a case where the LoF concern can be justified instrumentally. With asymmetric information as in our model, in many types of dynamic matching and sorting/screening games, an individual's willingness to accept another person might be taken by others as a negative signal of her type. This may harm her directly - through reduced esteem, as in Bernheim (1994). It may also harm her future material payoffs, particularly if the other party rejected her, so she must continue searching for a mate. To reinterpret Groucho Marx "if I am willing to be part of this club, how good can $I$ be?" We give a simple formalization of this in a two-period model in appendix C.7, where we derive conditions under which a players' previous "accept" choice hurts her continuation value.

Our theory is supported by evidence from psychology, sociology, and the professional matchmaking industry. There is abundant psychological evidence that "rejection hurts" (Eisenberger and Lieberman, 2004) and that social ostracism can cause a neurochemical effect that resembles physical pain (Williams, 2007). ${ }^{5}$

Our key assumption - that people suffer when their 'accept' decision is observed by someone who rejects them - is supported by previous work on romantic relationship initiation, which finds that people strive to avoid revealing unrequited interest, dislike the prospect of rejection, and suffer a loss of reputation from making too many offers. Douglas (1987) reports eight strategies that people use to learn whether those potential romantic partners like them, while Baxter and Wilmot (1984) described six types of secret tests (e.g., "third-party tests"). Shanteau and Nagy (1979) found that people were uninterested in pursuing a relationship when the probability of acceptance was low. In Eastwick and Finkel's (2008) speed-dating experiment, participants "who desired everyone were perceived as likely to say yes to a large percentage of their speed-dates [which] negatively predicted their desirability" (pp. 225). ${ }^{6}$

The fear of LoF is closely related to rejection sensitivity. E.g., peer rejection among middleschool students has been found to predict an increase in late "anxious and angry expectations", which are positively correlated to later social anxiety, social withdrawal, and loneliness (London

Betty's harmful rejection by rejecting her back. Note thatLoF has no clear fairness/reciprocity interpretation as in (Rabin, 1993): as revelation occurs after choices are made, these shouldn't affect beliefs about the congruence of players' intentions (kindness/unkindness).

${ }^{4}$ If reputation concerns are long-term, anticipating the additional short-run pain of losing face may help counteract present-bias as well as overconfidence.

${ }^{5}$ However, these studies do not distinguish private versus public 'accept' choices; there is scope for further observational and experimental work.

${ }^{6}$ While there are alternative explanations for some of these observations, these are consistent with our story of inherent and reputation-based LoF. 
et al, 2007, abstract). The authors argue that the rejection sensitive may exhibit "behavioral overreactions" such as "'flight' (social anxiety/withdrawal) or 'fight' (aggression)." Either of these can be interpreted as a way to pre-emptively choose "reject" (as in our matching game) to avoid further loss of face. This is also consistent with Goffman's (2005) discussion of preserving face through avoidance behavior and the use of go-betweens.

Speed dating can be seen as a canonical example of an institution designed to minimize LoF, and they promote themselves on these grounds. ${ }^{7}$ In speed dating events, participants meet in person to spend a few minutes in conversation with each of 20 or so singles of the opposite sex. Speed daters are usually asked to select whom they would like to go on "real dates" with only after the event, and most agencies only reveal proposals where there is a mutual match, i.e., both participants have selected each other. ${ }^{8}$

For the rejection-sensitive, any economic transaction that involves an "ask" may risk a LoF. This may explain the prevalence of posted prices, aversion to bargaining in certain countries, and the relative absence of neighborhood cooperation, social interaction, consumption and tasksharing in many modern societies (Putnam, 1995). Rejection sensitivity is particularly disabling for sales personnel who suffer from "call reluctance" (Geery, 1996). In the conclusion, we discuss several additional environments - including online dating, the job market, minority outreach, and international negotiations - in which LoF seems to play an important role, and where changes in policy, technology, and business practice may improve outcomes.

While economists have previously studied related concepts, to our knowledge none have considered the difference between "mutually-observed acceptance and rejection" and "rejection where only one side knows he was rejected" (and the other side does not know whether or not she was proposed to). Becker (1973) introduced a model of equilibrium matching in his "Theory of Marriage." He considers the surplus generated from marriage through a household production function, and allows the division of output between spouses to be divided ex-ante according to each party's outside option in an efficient "marriage market." Anderson and Smith (2010) brought reputation into this context, noting that matches yield both output and information about types (however, offers are not observed in their model). Chade (2006) explored a search and matching environment where participants observe noisy signals of their partners' types. He notes an "acceptance curse" resembling the winner's curse from auction theory: a partner's acceptance decision reveals information about their type. However, Chade's model only allows a single interaction between the same man and woman, and outside parties do not observe the results; thus there is no scope for either party's actions to affect their future reputations, nor any direct cost of being rejected.

Our work shares some features of standard models of directed search (e.g., Albrecht et al., 2006), but there are important differences. In previous employment models, the cost of locating an opening and submitting a job application (a "search friction") is unrelated to the probability of success, as well as unrelated to whether the employer knows a candidate has applied. In our model (as applied to this example) the job seeker knows that if there is a high probability they will get the job (even if they do not accept the offer), there is a low probability they will lose face, and thus this cost is low in expectation.

\footnotetext{
${ }^{7}$ E.g., Xpress dating advertises "rejection free dating in a non-pressurized environment" <http://www.xpressdating.co.uk/speed_intro.htm>,accessed November 2012.

8 "Speed dating" institutions have been extended outside the realm of romance and marriage, into forming study groups, "speed networking", "industrial speed dating," and business partnering; these may have been established (in part) to minimize LoF. See, e.g., Collins and Goyder (2013), CNN International (2005).
} 
Our paper proceeds as follows. In section 2 we describe our baseline setup, yielding only monotonic equilibria, following the theory of games with strategic complementarities (summarized in Vives, 2005). In section 3 we introduce LoF on one side to the model, and illustrate how this can cause a welfare loss, and how anonymity can improve this outcome. In particular, LoF can make low types "reverse snobs", rejecting only after observing higher signals. LoF also makes a coordination-failure equilibrium - where high types always reject - stable. We consider monotone comparative statics as a small amount of $\mathrm{LoF}$ is introduced to the environment (an application of Milgrom and Shannon, 1994): for any best-reply stable interior equilibrium, this makes high types more selective. We conclude in section 4, considering extensions and discussing policy implications. Longer and more technical proofs are in the appendix, as well as a model illustrating how reputation concerns in a two-stage game may resemble intrinsic LoF.

\section{Setup and benchmark model}

\subsection{Agents}

The economy is populated by a continuum of individuals on market sides $M$ and $F$ (for "male" and "Female") endowed with measure 1 each. An individual $m \in M$ or $f \in F$ is characterized by a binary type $x_{j} \in\{\ell, h\}$ (for "high" and "low") where $j \in\{m, f\}$; the type is an agent's private information. ${ }^{9}$ Let the share of $h$ types be the same on both sides of the market, and denote it by $p .{ }^{10}$

\subsection{Matching and information structure}

At the beginning of the game, each individual in $M$ is randomly matched to a single individual in $F$; all matches are chosen by nature with equal probability. Next, person $i$ obtains a noisy signal $s_{j} \in[\underline{s}, \bar{s}]$ (signals are independently chosen by nature) about the type $x_{j}$ of her match $j$, but does not observe $s_{i}$, the signal of her own type that $j$ received. After observing the signals, individuals accept $(A)$ or reject $(R)$ the match.

Here, we suppose that acceptance decisions are made sequentially and let males move first. However, we display the payoffs in matrix form below. Note that simultaneous and sequential environments will yield equivalent equilibrium results; we give intuition for this throughout, and show this in appendix B.

We distinguish two different informational settings:

(i) an Asymmetric Revelation Environment (ARE) where females observe the action $A$ or $R$ taken by a male but males do not observe females' actions - neither when they make their choices nor at the end of the game (however, males can infer female's actions from their own payoffs in some contingencies), and

(ii) a Conditionally Anonymous Environment (CAE) where neither side directly observes the others action (neither when making their choice nor at the end of the game), but only observe whether or not both parties have played accept. We compare the CAE to an Asymmetric

\footnotetext{
${ }^{9}$ In a previous draft (Hugh-Jones and Reinstein, 2010) we derived similar results with continuous types under certain functional restrictions. Note that our previous discussion also encompasses one-sided matching; we model a two-sided market (labeled "male" and "female" with apologies for political incorrectness) both because it is relevant to many examples and because it allows us to isolate both direct and indirect effects of a fear of LoF on one side.

${ }^{10}$ This assumption is made for notational simplicity, allowing that the type distributions differ by gender does not affect our results qualitatively.
} 
Revelation Environment as the one-way loss of face is easier to model and yields clearer intuition; This is further justified below. That is, in the CAE both males and females can infer their match's actions (proposals) if and only if they themselves play accept. ${ }^{11}$

\subsection{Payoffs}

If both individuals in a matched pair accept they become "married" and each individual's payoff depends positively on the pizzazz (see Burdett and Coles, 2006) of their partner: $\tilde{x} \in\{\tilde{\ell}, \tilde{h}\} . \ell$ types have pizzazz $\tilde{\ell}$ and $h$ types have pizzazz $\tilde{h}$, where $0<\tilde{\ell}<\tilde{h}$. Types (and thus pizzazz) become fully observable during the marriage. Marriage payoffs for a match $(m, f)$ are given by $u_{m}\left(\tilde{x}_{f}\right)=\tilde{x}_{f}$ and $u_{f}\left(\tilde{x}_{m}\right)=\tilde{x}_{m}$. That is, payoffs are linear in the match's type, which implies that total surplus does not depend on the precise assignment of types, but only on the number of marriages formed. ${ }^{12}$ Agents who remain solitary obtain a payoff equal to a share of their own pizzazz, i.e., $u_{j}\left(\tilde{x}_{j}\right)=\delta \tilde{x}_{j}$ for $j \in\{m, f\}$ with $\delta<1$. For intuition, suppose types represent productivity, production is shared by the married couple, and those who are more productive alone are also more productive in a marriage. ${ }^{13}$ Therefore $\ell$ types always prefer a marriage to remaining alone. To make the model non-trivial, we suppose that $h$ 's prefer to remain unmarried to marrying an $\ell$, i.e., high types have a good outside option:

Assumption 1. $\delta \tilde{h}>\tilde{\ell}$.

In summary, homogenous marriages benefit both partners and mixed marriages benefit $\ell$ 's more than they hurt $h$ 's. This follows from the larger total payoff, as $\tilde{h}+\tilde{\ell}>\delta(\tilde{h}+\tilde{\ell})$. Thus, the payoff matrix of the game played by a randomly matched pair $(m, f)$ is the following.

\begin{tabular}{l|ll}
$f^{m}$ & $A$ & $\mathrm{R}$ \\
\hline$A$ & $\tilde{x}_{m}, \tilde{x}_{f}$ & $\delta \tilde{x}_{m}, \delta \tilde{x}_{f}$ \\
$R$ & $\delta \tilde{x}_{m}, \delta \tilde{x}_{f}$ & $\delta \tilde{x}_{m}, \delta \tilde{x}_{f}$
\end{tabular}

While our setting does not allow for a generic analysis of matching games, it captures a large set of interactions in matching environments where loss of face may be relevant. Our assumptions embody agreed-upon preferences over a partner's type - partners are better or worse along a single dimension, although this may be a reduction of several characteristics.

\subsection{Signal distributions}

Signal distributions depends on the type of the sender: type $x$ 's $(x \in\{\ell, h\})$ signal is distributed according to $F_{x}(s)$ with continuously differentiable density $f_{x}(s)$. Suppose that the signal is informative in the sense that $f_{\ell}(s)$ and $f_{h}(s)$ satisfy the monotone likelihood ratio property, i.e.,

\footnotetext{
${ }^{11}$ We could instead model simultaneous-move games: a CAE where neither choice is revealed unless both choose accept, and an ARE where the female's choice is only revealed to a male if he accepts, while the male's choice is always revealed to the female. This would be strategically-equivalent, as, in the sequential model, the secondmover will always prefer to reject after observing a rejection. This would only serve to make the notation more cumbersome. Furthermore, as noted elsewhere, the sequential ARE has a natural real-world analogue: without an intermediary, one party must typically make the first move.

${ }^{12}$ We see this assumption as a reasonable benchmark, representing the knife-edge between increasing and decreasing differences (in the two variables "own type" and "other's type"). Allowing super- or sub-modularities in the marriage production function may be interesting for future research.

${ }^{13}$ This assumption might also be justified through interpreting the payoff to no match as the continuation value in an indefinitely repeated version of the matching game, as in, e.g., Adachi (2003), or as in our two-period model in appendix section C.7. In Chade (2006) this value increases in type as higher-type players tend to have higher signals and other players accept them more often.
} 
Assumption 2. $f_{h}(s) / f_{\ell}(s)>f_{h}\left(s^{\prime}\right) / f_{\ell}\left(s^{\prime}\right)$ for all $s>s^{\prime}$ where defined.

We assume that signals have limit distributions, i.e., that observing the best (worst) signal implies that the type is $h(\ell)$, i.e.,

Assumption 3. $f_{h}(\underline{s})=0, f_{\ell}(\underline{s})>0, f_{\ell}(\bar{s})=0$, and $f_{h}(\bar{s})>0$.

Assuming that the probability of a high (low) type converges to one (zero) will ensure that game has an interior equilibrium (signal thresholds for accepting a match will be interior), and it is crucial for lemma 1 , as further discussed below. ${ }^{14}$

We will look for Perfect Bayesian Equilibria and consider best-reply stability, i.e., stability with respect to iterative responses to deviations or "cobweb dynamics" (see Hahn, 1962, Dixit, 1986, and Vives, 2005). The stability results for corner-solution (coordination failure) equilibria will also hold under the trembling-hand perfection refinement (Selten, 1975).

\subsection{Benchmark model: no loss of face}

To illustrate the setup we now solve for the equilibrium behavior in the matching game without loss of face, which is equivalent to the CAE. Since $\delta<1$, low types always prefer any marriage to remaining alone. Therefore low types find it always (at least weakly) optimal to accept.

In a stable equilibrium low types must always accept with some minimum probability $\varepsilon>0$, which in turn, given assumption 3, implies that high types must at least sometimes reject. ${ }^{15}$ This implies that the acceptance behavior of players of type $h$ and $\ell$ differs in equilibrium, implying that the event of being accepted also conveys some information about the match's type (the "acceptance curse" in Chade, 2006). Let $q_{j}\left(x ; x^{\prime}\right)$ for $j \in\{m, f\}$ denote the resulting equilibrium probability that a type $x$ on side $j$ accepts a match, given the match is of type $x^{\prime}$. Note that this expression incorporates the acceptance curse as high types accept less often, i.e., $q(h ; h)<1$, thus being accepted implies a greater likelihood one's match is a low type. Since $\ell$ types always find it profitable to accept, $q_{j}\left(\ell, x^{\prime}\right)=1$ for $x^{\prime} \in\{\ell, h\}$ and $j \in\{m, f\}$.

We next consider a high type's response. For an individual $i$ of type $h$ in a match $(i, j)$, playing $R$ yields a payoff $\delta \tilde{h}$, whereas playing $A$ either yields $\tilde{x_{j}}$, if $j$ accepted, or $\delta \tilde{h}$ if $j$ rejected. That is, the decision to accept or reject only affects the payoff in the case that the match accepted. High types of both genders (whether or not they observed their match's action) find it (weakly) profitable to accept after observing a signal $s$ if and only if the expected payoff from accepting meets or exceeds the outside option:

$$
\frac{p f_{h}(s)}{(1-p) f_{\ell}(s)+p f_{h}(s)}\left[q_{j}(h, h) \tilde{h}+\left(1-q_{j}(h, h)\right) \delta \tilde{h}\right]+\frac{(1-p) f_{\ell}(s)}{(1-p) f_{\ell}(s)+p f_{h}(s)} \tilde{\ell} \geq \delta \tilde{h},
$$

\footnotetext{
${ }^{14}$ Alternatively, we could consider the alternate assumption of overlapping supports; i.e., for all $s \in[\underline{s}, \bar{s}]$ $f_{h}(s)>0$ if and only if $f_{\ell}(s)>0$. This alternative assumption would imply that we could not rule out equilibria where high types do not respond to the signal and instead "always accept" or "always reject," and these could be stable. However, even under overlapping supports, if we were to focus on "responsive" equilibria where high types have interior thresholds, our remaining results carry over. Details are available by request.

${ }^{15}$ Although there is always a "trivial coordination failure" equilibrium where both players always reject (since if $i$ 's match rejects with certainty, then either accepting or rejecting yield payoff $\delta \tilde{x}_{i}$ ), this equilibrium is neither best-reply-stable nor trembling-hand perfect. From such a point, if low (or high) types reduce their cutpoint slightly or tremble, thus playing accept with a positive probability, other low types would strictly prefer to play accept, i.e., set their cutpoint at $\underline{s}$. Individuals do not observe the signals of their own type. Thus, whether the other side reduces their cutpoint (accepting those with "nearly the highest" signal) or trembles randomly (randomly playing accept with a small probability), low types will strictly prefer to play accept. Note that this "trivial coordination failure" is distinct from the case where low types always accept and high types always reject, which we call the Coordination Failure Equilibrium or "C-F equilibrium"; we return to this below.
} 
where $j=m, f$. If we subtract $\delta \tilde{h}$ from both sides of the above inequality and rearrange terms, we see that an $h$ accepts if the gain from marrying another $h$ outweighs the loss from marrying an $\ell$ in expectation conditional on the signal and considering equilibrium play, i.e., if: ${ }^{16}$

$$
p f_{h}(s)\left[q_{j}(h, h) \tilde{h}+\left(1-q_{j}(h, h)\right) \delta \tilde{h}-\delta \tilde{h}\right] \geq(1-p) f_{\ell}(s)(\delta \tilde{h}-\tilde{\ell}) .
$$

Note that the overall probability of being accepted as a function of types, $q_{j}\left(x^{\prime} ; x\right)$ does not depend on the signal $s$ that the player observes, as signals are drawn independently and individuals do not observe the signals of their own type.

Because of the monotone likelihood property, (2) implies that an agent $i$ of type $h$ accepts if and only if the signal $s$ that $i$ observes is sufficiently high. Hence, there is $\hat{s} \in[\underline{s}, \bar{s}]$ such that an agent of type $h$ finds it profitable to accept if and only if he observes $s \geq \hat{s}$. That is, both males and females use threshold strategies, accepting only if the signal exceeds their threshold, $\hat{s}_{m}$ and $\hat{s}_{f}$ respectively. This implies that an agent $j \in\{m, f\}$ of type $h$ accepts an agent of type $x$ with probability

$$
q_{j}(h, x)=1-F_{x}\left(\hat{s}_{j}\right), \text { with } j \in\{m, f\} .
$$

Using this on (2) implies that, given a cutoff value $\hat{s}_{j}$ on the other market side, a high type's optimal reply is to accept if

$$
\frac{f_{h}(s)}{f_{\ell}(s)} \geq \frac{1-p}{p} \frac{\delta \tilde{h}-\tilde{\ell}}{(1-\delta) \tilde{h}} \frac{1}{1-F_{h}\left(\hat{s}_{j}\right)}
$$

and to reject otherwise. We can thus define the high types' best reply in any stable or trembling-hand perfect equilibrium: accept if and only if $s \geq \hat{s}_{i}^{*}\left(\hat{s}_{j}\right)$, where $i \neq j \in\{m ; f\}$ and

$$
\hat{s}_{i}^{*}\left(\hat{s}_{j}\right): \frac{f_{h}\left(\hat{s}_{i}^{*}\right)}{f_{\ell}\left(\hat{s}_{i}^{*}\right)}=\frac{1-p}{p} \frac{\delta \tilde{h}-\tilde{\ell}}{(1-\delta) \tilde{h}} \frac{1}{1-F_{h}\left(\hat{s}_{j}\right)} .
$$

Continuity of the signal and its distribution and the monotone likelihood property ensure that the best response $\hat{s}_{i}^{*}($.$) exists and that \hat{s}_{i}^{*}($.$) weakly increases in its argument. The latter$ implies that thresholds are strategic complements. Combined with the symmetry of the game in types this implies that $\hat{s}_{m}=\hat{s}_{f}:=\hat{s}^{*}$ in a Nash equilibrium (supposing the contrary leads quickly to a contradiction). As noted above, $\hat{s}^{*}>\underline{s}$ in any stable equilibrium. Since agents' actions do not affect other agents' information sets, beliefs are always formed according to Bayes' rule, and the issue of out-of-equilibrium beliefs will not arise.

Totally differentiating and rearranging terms (see footnote) we see the slope of a high-type player i's best response function must follow: ${ }^{17}$

$$
\frac{\partial \hat{s}_{i}}{\partial \hat{s}_{j}}=\frac{f_{h}\left(\hat{s}_{i}\right) f_{h}\left(\hat{s}_{j}\right)}{\frac{\partial f_{h}\left(\hat{s}_{i}\right)}{\partial s}\left(1-F_{h}\left(\hat{s}_{j}\right)\right)-\frac{1-p}{p} \frac{\delta \tilde{h}-\tilde{\ell}}{(1-\delta) \hat{h}} \frac{\partial f_{\ell}\left(\hat{s}_{i}\right)}{\partial s}} .
$$

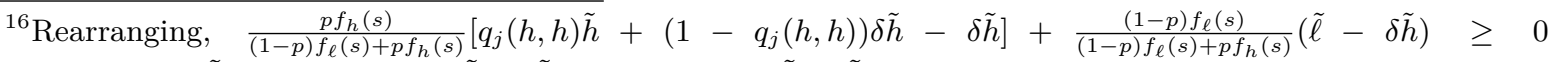
$\Leftrightarrow p f_{h}(s)\left[q_{j}(h, h) \tilde{h}+\left(1-q_{j}(h, h)\right) \delta \tilde{h}-\delta \tilde{h}\right]+(1-p) f_{\ell}(s)(\tilde{\ell}-\delta \tilde{h}) \geq 0, \Leftrightarrow$ inequality 3 .

${ }^{17}$ Rearranging equation 5 , $i$ 's optimal cutpoint $\hat{s}_{i}^{*}\left(\hat{s}_{j}\right)$ is defined by $f_{h}\left(\hat{s}_{i}^{*}\right)\left(1-F_{h}\left(\hat{s}_{j}\right)\right)=\frac{1-p}{p} \frac{\delta \tilde{h}-\tilde{\ell}}{(1-\delta) \tilde{h}} f_{\ell}\left(\hat{s}_{i}^{*}\right)$. Totally differentiating yields: $\frac{\partial f_{h}\left(\hat{s}_{i}^{*}\right)}{\partial s}\left(1-F_{h}\left(\hat{s}_{j}\right)\right) \Delta \hat{s}_{i}^{*}-f_{h}\left(\hat{s}_{i}^{*}\right) f_{h}\left(\hat{s}_{j}\right) \Delta \hat{s}_{j}=\frac{1-p}{p} \frac{\delta \tilde{h}-\tilde{\ell}}{(1-\delta)} \frac{\partial f_{\ell}\left(\hat{s}_{i}^{*}\right)}{\partial s} \Delta \hat{s}_{i}^{*}$. Rearranging yields: $\left(\frac{\partial f_{h}\left(\hat{s}_{i}^{*}\right)}{\partial s}\left(1-F_{h}\left(\hat{s}_{j}\right)\right)-\frac{1-p}{p} \frac{\delta \tilde{h}-\tilde{\ell}}{(1-\delta) \hat{h}} \frac{\partial f_{\ell}\left(\hat{s}_{i}^{*}\right)}{\partial s}\right) \Delta \hat{s}_{i}^{*}=f_{h}\left(\hat{s}_{i}^{*}\right) f_{h}\left(\hat{s}_{j}\right) \Delta \hat{s}_{j}$. The usual argument then yields differentiability of the best response function and the slope of the reaction function in equation 6 .
} 
A sufficient condition for an interior stable equilibrium to exist is

$$
\left(f_{h}(\bar{s})\right)^{2}>-\frac{\partial f_{\ell}(\bar{s})}{\partial s} \frac{1-p}{p} \frac{\delta \tilde{h}-\tilde{\ell}}{(1-\delta) \tilde{h}}
$$

Condition 7 ensures that that a high type's reaction function has a slope greater than unity at $\bar{s}$ (which also implies that the C-F equilibrium is not stable). Condition 7 would be implied by $\frac{\partial f_{\ell}(\bar{s})}{\partial s}=0$, i.e., if the low type's signal distribution becomes flat at $\bar{s} .{ }^{18}$ Condition 7 will also hold if the underlying share of high types is sufficiently great; e.g., for a linear density on $[0,1]$ condition 7 holds if $p>\frac{\delta \tilde{h}-\tilde{\ell}}{2 \tilde{h}(1-\delta)+\delta \tilde{h}-\tilde{\ell}}$.

Note that the slope of the best response function (6) must be zero at $\hat{s}_{j}=\underline{s}$ because of the continuous limit distributions $\left(f_{h}(\underline{s})=0\right)$. Further recalling $\hat{s}>\underline{s}$, we see that if condition 7 holds, then the best response function must intersect the 45 degree line at least once, so there will an interior equilibrium, as shown in Figure 1. Since the best response function is increasing, at least one intersection point will have a slope below one and thus correspond to a stable equilibrium. For a unique equilibrium we would further require the best response function to be convex. If condition 7 does not hold then the C-F equilibrium will be stable, and we may not have an interior equilibrium.

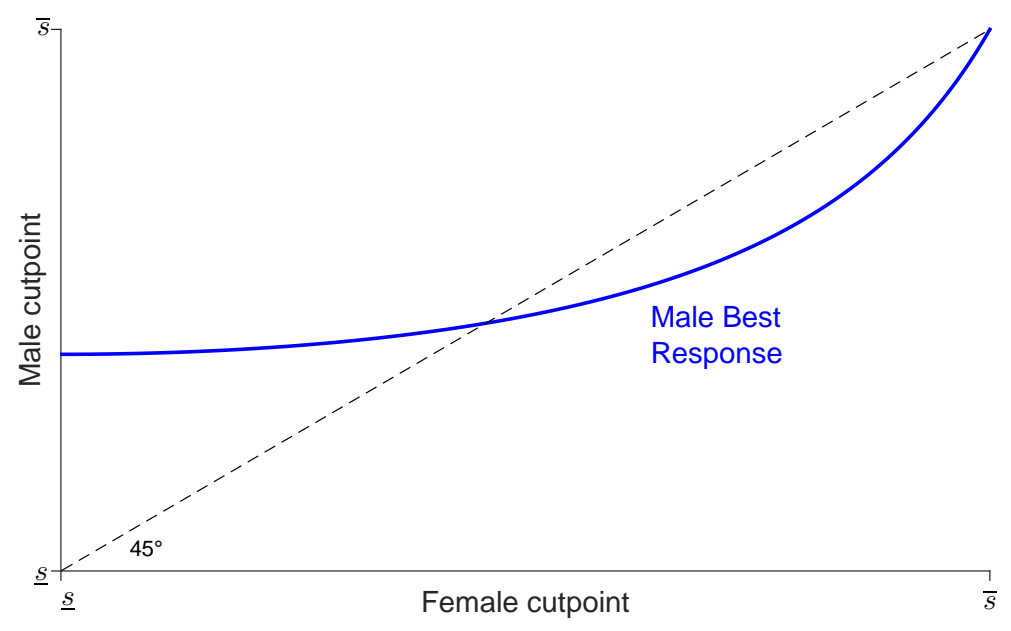

Figure 1: Male high type's best response to high type female cutpoints; condition 7 holds here

We summarize the above results for the game without loss of face in the following lemma.

Lemma 1. If condition 7 holds then at least one interior, stable equilibrium exists, and in any stable equilibrium

1. low types always accept,

2. high types use symmetric cutoff strategies, accepting if $s>\hat{s}_{m}=\hat{s}_{f}:=\hat{s}^{*}$, and

3. $\hat{s}^{*} \in(\underline{s}, \bar{s})$.

Simple calculations yield a corollary to this result:

\footnotetext{
${ }^{18}$ The flat signal distribution would also ensure that the C-F equilibrium is not trembling-hand perfect, since any tremble would make it a dominant strategy for a high type to accept against any signal that cannot come from a low type.
} 
Corollary. Where condition 7 holds, expected payoffs for types $\ell$ and $h$ in a stable equilibrium of the game without loss of face (or for any strategy profile where low types always accept) are

$$
\begin{aligned}
& v(\ell)=\underbrace{\delta \tilde{\ell}}_{\text {single } \ell}+\underbrace{p\left(1-F_{\ell}\left(\hat{s}^{*}\right)\right)(\tilde{h}-\delta \tilde{\ell})}_{\ell \text { 'fools' } h}+\underbrace{(1-p)(\tilde{\ell}-\delta \tilde{\ell})}_{\ell \text { marries } \ell} \text { and } \\
& v(h)=\underbrace{\delta \tilde{h}}_{\text {single } h}+\underbrace{p\left(1-F_{h}\left(\hat{s}^{*}\right)\right)^{2}(\tilde{h}-\delta \tilde{h})}_{h \text { marries } h}-\underbrace{(1-p)\left(1-F_{\ell}(\hat{s})\right)(\delta \tilde{h}-\tilde{\ell})}_{h \text { 'fooled by' } \ell} .
\end{aligned}
$$

Note $v(h)>v(\ell)$. The number of marriages is $(1-p)^{2}+2 p(1-p)\left(1-F_{\ell}\left(\hat{s}^{*}\right)\right)+p^{2}\left(1-F_{h}\left(\hat{s}^{*}\right)\right)^{2}$, which strictly decreases in $\hat{s}^{*}$.

The above payoffs sum up the following intuition. An $\ell$ will not marry (and will thus get $\delta \tilde{\ell}$ ) unless he fools an $h$ or meets another $\ell$. An $h$ will marry only if he meets another $h$ and they both send very positive signals, or if he is fooled by an $\ell$ (i.e., he meets an $\ell$ who sends a high enough signal).

In the above game without loss of face the order of play does not affect outcomes. Since both players' actions impact payoffs only in the case that the other player plays accept, their best responses do not change if they learn whether or not the other player did in fact accept; the same acceptance curse occurs in either case.

\section{Intrinsic Loss of Face in an Asymmetric Revelation Environ- ment}

Loss of face as described in section 1 is an intrinsic psychological pain, which can only matter if a player's potentially embarrassing action is observed by the other player. Therefore we define loss of face as follows.

Definition 1. A player $j$ who suffers from intrinsic loss of face suffers a loss $L$ when

1. j played accept.

2. his match, player $k$, played reject, and

3. $k$ knows (for certain) that $j$ played accept, and

4. $j$ knows all of the above.

The final point may be necessary for a primal LoF (but see Battigalli and Dufwenberg, 2009 on the equivalency of second and first-order beliefs in these models) but not for the reputational LoF we model in Appendix C.7; my reputation and future payoffs may suffer whether or not I know that my decision is observed. As we discuss further below, we can also relax point 3: our equilibrium predictions are robust to allowing LoF to be a continuous function of the probability with which $k$ believes that $j$ played "accept", when $k$ plays reject; best responses would not change (see Appendix C.2 for a full proof).

Since LoF results from the mutual knowledge of one party accepting and the other rejecting, to model LoF we need to make payoffs depend not only on actions, but also on the information players hold at the end of the game. These terminal information sets for players $m$ and $f$ are defined as standard information sets, but they are not at a decision node: they characterize a player's knowledge about the complete history of the game after all actions have been taken. 
In the game defined above terminal information sets will depend on the information environment in place (this is graphically depicted in figure 3 in the appendix). Denote an action tuple by $\left(a_{m}, a_{f}\right) \in\{A ; R\}^{2}$. If both players in a match only observe their own actions and whether or not there is a marriage (the conditionally anonymous environment, CAE), both players' information sets are $\{\langle A, A\rangle\},\{\langle R, A\rangle,\langle A, R\rangle\}$, or $\{\langle A, R\rangle\}$. This implies that females cannot distinguish between action profiles $(A, R)$ and $(R, R)$, and males cannot distinguish between $(R, A)$ and $(R, R)$. Therefore there is no loss of face under the CAE, and equilibrium behavior under the CAE is given as in the previous section (see especially lemma 1 and its corollary).

As noted earlier, having only one side vulnerable to LoF will allow us to separately consider both direct and indirect effects, and will be relevant to the comparison between sequential decision environments and the CAE. Here we will assume that the males move first; i.e., consider "males" as synonymous with "first-movers" or "the side vulnerable to LoF."

If females observe males' actions in a match, but not vice versa (the asymmetric revelation environment, ARE), possible terminal information sets are $\{\langle R, A\rangle\},\{\langle R, R\rangle\},\{\langle A, A\rangle\}$, or $\{\langle A, R\rangle\}$ for females, and $\{\langle A, A\rangle\},\{\langle A, R\rangle\}$, and $\{\langle R, A\rangle,\langle R, R\rangle\}$ for males. ${ }^{19}$ That is, it is common knowledge that females observe the action profile $(A, R)$, whereas males cannot distinguish $(R, A)$ and $(R, R)$. Therefore there is loss of face for males when the action profile $(A, R)$ is played, but there is no loss of face for females under the ARE. ${ }^{20}$

Under the ARE the payoff matrix (1) changes into:

\begin{tabular}{l|ll}
$m^{f}$ & $A$ & $R$ \\
\hline$A$ & $\tilde{x}_{f}, \tilde{x}_{m}$ & $\delta \tilde{x}_{f}-L, \delta \tilde{x}_{f}$ \\
$R$ & $\delta \tilde{x}_{f}, \delta \tilde{x}_{m}$ & $\delta \tilde{x}_{f}, \delta \tilde{x}_{m}$
\end{tabular}.

As above, individuals' acceptance decisions will depend on the inference they make about their match's type given the signal and given the event of being accepted. Because only males may incur loss of face, the decision problems of males and females now differ.

Note that Definition 1 could be generalized to allow $j$ 's LoF term $L$ to be a continuous nondecreasing function of the probability that $k$ puts on $j$ having played accept. This would have no impact on best-response functions, implying that all of our results below would be preserved exactly conditional on $L$, which would be an equilibrium quantity. ${ }^{21}$

\subsection{Female behavior in the ARE}

We begin with the females' decision. Since females do not suffer from loss of face the analysis from section 2.5 carries over: $\delta>0$ implies again that low-type females find it always profitable to accept. As before, denote by $q_{m}(h, h)$ and $q_{m}(\ell, h)$ the probabilities that male $h$ and $\ell$ types, respectively, accept female $h$ types in equilibrium (and $q_{f}$ is defined similarly for females

\footnotetext{
${ }^{19}$ Examining the remaining two possible informational environments is straightforward and does not yield any additional insights.

${ }^{20}$ Equivalently, even if both females and males had four terminal information sets, if the males choose first and the females observe this and choose second, a female would never accept unless the male did, and thus the outcomes would be as if she were not vulnerable to LoF.

${ }^{21}$ The equilibrium probability a randomly matched $j$ plays accept (conditional on the $k$ 's type) is an aggregate of a continuum of players; hence it is unaffected by any $j$ 's strategy. Thus, for this alternate assumption, the exact LoF cost would depend on equilibrium play (and type distributions), but equilibrium strategies would take the LoF term as an exogenous constant. This would lead to a decrease in payoffs for both sides in the CAE, assuming that the maximal $\mathrm{LoF}$, where $k$ believes that $j$ played accept with probability 1 , is $L$, the same term as above. However, best responses would be unchanged. (see Appendix C.2 for a full proof).
} 
accepting males); again, this allows an acceptance curse. Female $h$ types find accepting profitable if, given the observed signal $s$, the expected payoff from accepting exceeds the outside option (noting that the payoff from accepting only differs from the outside option where the male accepts, and the probability he accepts depends on his type). Thus, restating inequality 2 for females, and allowing low males to reject with positive probability, she will accept if and only if:

$$
\begin{aligned}
& \underbrace{\frac{p f_{h}(s)}{(1-p) f_{\ell}(s)+p f_{h}(s)}}_{\operatorname{Pr}(\text { high male }) \text { signal } s}[\underbrace{q_{m}(h, h)}_{\operatorname{Pr}(\mathrm{h} \text { male accepts h fem })} \tilde{h}+\underbrace{\left(1-q_{m}(h, h)\right)}_{\operatorname{Pr}(\mathrm{h} \text { male rejects h fem })} \delta \tilde{h}] \\
& +\frac{(1-p) f_{\ell}(s)}{\underbrace{(1-p) f_{\ell}(s)+p f_{h}(s)}_{\text {Pr(low male) })}}[\underbrace{q_{m}(\ell, h)}_{\operatorname{Pr}(1 \text { male accepts h fem })} \tilde{\ell}+\underbrace{\left(1-q_{m}(l, h)\right)}_{\operatorname{Pr}(1 \text { male rejects h fem })} \delta \tilde{h}] \\
& \geq \delta \tilde{h} .
\end{aligned}
$$

Since $q_{m}(h, h)$ and $q_{m}(\ell, h)$ do not depend on the signal that the female player observes, the monotone likelihood property implies, as in the benchmark model, that a female of type $h$ must have a single threshold cutoff. As the first bracketed term strictly exceeds the second bracketed term, ${ }^{22}$, the female payoff to accepting must increase in the probability the male is a high type. Thus the female cutoff must again be a floor, i.e., accept the signal $s \geq \hat{s}$ for some threshold $\hat{s} \in[\underline{s}, \bar{s}]$.

\subsection{Male behavior in the ARE}

Given female behavior (threshold floor $\hat{s}_{f}$ for high females, low females always accept), males of type $x$ who observe a signal $s$ find it profitable to accept if and only if the expected payoff from accepting, including the expected loss of face when rejected, exceeds the outside option:

$$
\begin{aligned}
& \underbrace{\frac{(1-p) f_{\ell}(s)}{(1-p) f_{\ell}(s)+p f_{h}(s)}}_{\operatorname{Pr}\left(x_{f}=\ell \mid s\right)} \tilde{\ell}+ \\
& \underbrace{\frac{p f_{h}(s)}{(1-p) f_{\ell}(s)+p f_{h}(s)}}_{\operatorname{Pr}\left(x_{f}=h \mid s\right)} \times[\underbrace{\left(1-F_{x}\left(\hat{s}_{f}\right)\right) \tilde{h}}_{\operatorname{Pr}(h \text { fem. accepts }) \times \text { marr. util. } \quad}+\underbrace{F_{x}\left(\hat{s}_{f}\right)(\delta \tilde{x}-L)}_{\operatorname{Pr}(h \text { fem. rejects }) \times \text { reject. util. }}] \geq \delta \tilde{x} .
\end{aligned}
$$

Collecting terms, a male of type $x$ accepts if

$$
(1-p) f_{\ell}(s)(\tilde{\ell}-\delta \tilde{x})+p f_{h}(s)\left[F_{x}\left(\hat{s}_{f}\right)(\delta \tilde{x}-L-\tilde{h})+\tilde{h}-\delta \tilde{x}\right] \geq 0
$$

Stating this condition in terms of likelihood ratios we have

$$
\frac{1-p}{p} \frac{f_{\ell}(s)}{f_{h}(s)}(\tilde{\ell}-\delta \tilde{x})-F_{x}\left(\hat{s}_{f}\right)(L+\tilde{h}-\delta \tilde{x})+\tilde{h}-\delta \tilde{x} \geq 0
$$

Now the monotone likelihood ratio property implies that the left-hand side strictly decreases in $s$ for low type males and strictly increases in $s$ for high males, implying any cutoff thresholds

\footnotetext{
${ }^{22}$ If males always reject, this would be a weak inequality. However, this could not be part of a stable nor trembling-hand perfect equilibrium; low males and low females observing the lowest signals would naturally deviate.
} 
must be floors for high-type males and ceilings for low-type males. Furthermore, male low types accept with probability one if they would prefer to accept when matched with a high-type female (i.e., if the expected value of the marriage gain exceeds the expected loss of face from possible rejection), i.e.,

$$
\left(1-F_{\ell}\left(\hat{s}_{f}\right)\right)(\tilde{h}-\delta \tilde{\ell}) \geq F_{\ell}\left(\hat{s}_{f}\right) L,
$$

while male high types reject with probability one if they would prefer to reject even when matched with a high-type female, i.e.,

$$
\left(1-F_{h}\left(\hat{s}_{f}\right)\right)(\tilde{h}-\delta \tilde{h}) \leq F_{h}\left(\hat{s}_{f}\right) L
$$

This implies the following lemma, derived in the appendix.

Lemma 2. With loss of face in an ARE males use threshold strategies; $\ell$ types use ceilings, $h$ types use floors.

1. Male $h$ types play "accept iff $s_{f} \geq \hat{s}_{h}$ " with $\hat{s}_{h}=\bar{s}$ if (14) holds; otherwise $\hat{s}_{h}$ is interior and is defined by

$$
\frac{f_{h}\left(\hat{s}_{h}\right)}{f_{\ell}\left(\hat{s}_{h}\right)} \frac{p}{1-p} \frac{(1-\delta) \tilde{h}}{\delta \tilde{h}-\tilde{\ell}}=\frac{1}{1-F_{h}\left(\hat{s}_{f}\right)(1+L /((1-\delta) \tilde{h}))} .
$$

2. Male $\ell$ types play "accept iff $s_{f} \leq \hat{s}_{\ell}$ " with $\hat{s}_{\ell}=\bar{s}$ if (13) holds; otherwise $\hat{s}_{\ell}$ is interior and is defined by

$$
\frac{f_{h}\left(\hat{s}_{\ell}\right)}{f_{\ell}\left(\hat{s}_{\ell}\right)} \frac{p}{1-p} \frac{\tilde{h}-\delta \tilde{\ell}}{\delta \tilde{\ell}-\tilde{\ell}}=\frac{1}{1-F_{h}\left(\hat{s}_{f}\right)(1+L /(\tilde{h}-\delta \tilde{\ell}))} .
$$

3. Female low types always accept, while female high types play "accept iff $s_{m} \geq \hat{s}_{h}$ ", where $\hat{s}_{h}$ is defined by equation $10 .^{23}$

As in the benchmark case, male high types attempt to avoid marrying female low types and therefore screen for female high types using the signal. However, unlike the benchmark case, with sufficiently severe loss of face, male low types attempt to avoid being rejected and use the signal to screen for female low types. Thus loss of face can make low types behave as "reverse snobs", rejecting only those matches who send higher signals.

Figure 2 depicts a male high type's best response to a female high type's choice of cutpoint when an interior equilibrium exists. The presence of LoF shifts the male reaction function upwards, and thus generally makes male high types more conservative (i.e., requiring a higher signal) in accepting females. This change results in another intersection point of the best reply function with the $45^{\circ}$ line, which suggests that the set of equilibria may differ substantially, however. As we will see below, adding LoF may introduce a new, unstable equilibrium and render the previously unstable C-F equilibrium stable.

\footnotetext{
${ }^{23}$ We solve for this in equation 18 below. Note that where (13) holds (low males always accept), this is identical to the female's best response function without LoF.
} 


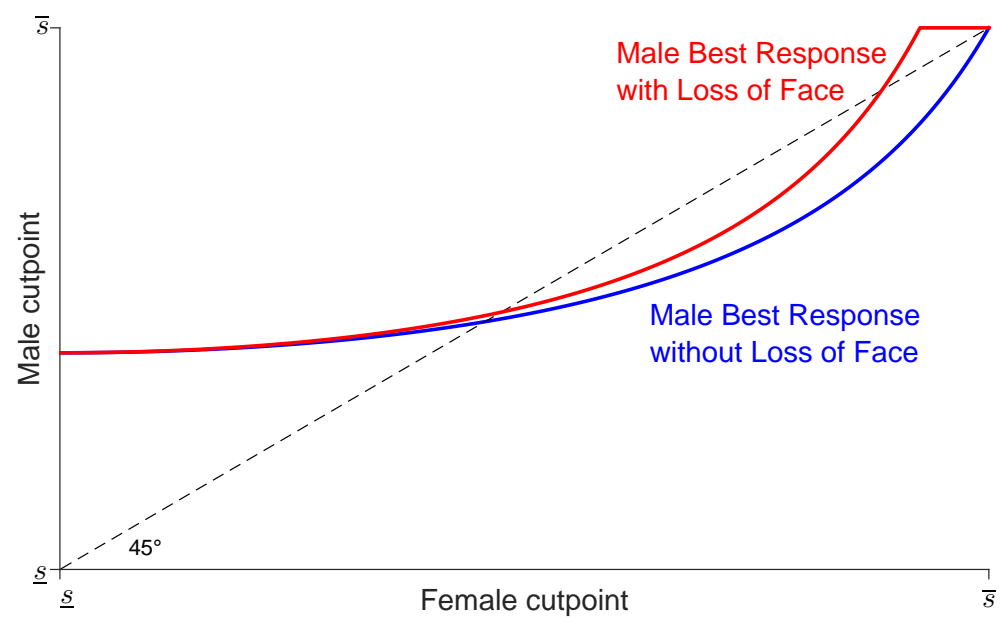

Figure 2: Male high-type's best response to (high type) female cutpoints, with and without loss of face; condition 7 holds here.

\subsection{Properties of an equilibrium in the ARE}

We focus here on the case where an interior equilibrium exists with or without LoF (implying that the C-F equilibrium is unstable without $\mathrm{LoF}$ ). Since males continue to use threshold strategies we can now pin down the high-type females' threshold $\hat{s}_{f}$ (recalling that female low types always accept). Allowing low type males to set a ceiling $\hat{s}_{\ell}$, after observing a signal $s$ a female high type accepts if and only if

$$
p\left(1-F_{h}\left(\hat{s}_{h}\right)\right) f_{h}(s)(\tilde{h}-\delta \tilde{h}) \geq(1-p) F_{h}\left(\hat{s}_{\ell}\right) f_{\ell}(s)(\delta \tilde{h}-\tilde{\ell}) .
$$

This implies that where

$$
\left(1-F_{h}\left(\hat{s}_{h}\right)\right)(\tilde{h}-\delta \tilde{h})<0,
$$

a female high type will never accept (i.e., $\hat{s}_{f}=\bar{s}$ ), which can only hold if male high types never accept, i.e., where $\hat{s}_{h}=\bar{s}$; i.e., this is the C-F equilibrium.

Otherwise her cutoff $\hat{s}_{f}$ will be interior and given by

$$
\frac{f_{h}\left(\hat{s}_{f}\right)}{f_{\ell}\left(\hat{s}_{f}\right)} \frac{p}{1-p} \frac{(1-\delta) \tilde{h}}{\delta \tilde{h}-\tilde{\ell}}=\frac{F_{h}\left(\hat{s}_{\ell}\right)}{1-F_{h}\left(\hat{s}_{h}\right)} .
$$

\section{Comparative statics}

We next consider how the equilibrium changes around the benchmark $L=0$, as we add a small loss of face term, $L>0$. For $L=0$, (the benchmark case) low types always accept, and an interior threshold $\left(\hat{s}^{*}\right)$ for female high types satisfies (5), that is,

$$
\frac{f_{h}\left(\hat{s}^{*}\right)}{f_{\ell}\left(\hat{s}^{*}\right)} \frac{p}{1-p} \frac{(1-\delta) \tilde{h}}{\delta \tilde{h}-\tilde{\ell}}=\frac{1}{1-F_{h}\left(\hat{s}^{*}\right)} .
$$

Perturbing this with a small $L>0$, we examine the behavior of the system of equations $(15,16$, and 18) in the neighborhood of $\hat{s}^{*}$. For a sufficiently small $L>0$ condition 13 holds, thus all low types accept, but the high types' optimal thresholds are perturbed. Noting this yields the following statement (proof in the appendix). 
Proposition 1. Suppose that $L=0$ and that there is an interior equilibrium. Here $\hat{s}^{*}$ will be implicitly defined by (19). Then, for a small increase in $L$ of $\epsilon>0$ low males still always accept, i.e. $\hat{s}_{\ell}=\bar{s}$, and the equilibrium cutoffs for high females and high males move in the same direction. For any interior equilibrium the cutoffs will increase if the local slopes of the reaction functions are below 1, i.e., in a stable equilibrium; they will decrease if these slopes exceed 1, i.e., in an unstable equilibrium.

For intuition, consider that for equilibrium dynamics, becoming more selective by increasing one's cutoff has a twofold effect on the expected quality of a marriage partner. First, there is a screening effect, increasing the expected quality of a match holding constant the acceptance behavior of the other gender. Second, there is a supply effect in the opposite direction: if one side becomes more selective, then the other side will react by also becoming more selective, implying a greater acceptance curse on both sides. ${ }^{24}$

The screening effect response to LoF is straightforward. Holding the females' cutoffs constant, the risk of LoF makes playing accept less attractive for males. Thus a high type male would require a greater expected payoff from marriage to get him to play accept, and thus he might raise his signal. This would make the female raise her signal as well (although as noted, in a stable equilibrium, by not as much).

However, if we restrict our consideration to stable equilibria, the counter-intuitive response can be ruled out. As noted in the above proposition, starting from any stable equilibrium, a small amount of LoF makes all high types more selective, i.e., $\hat{s}_{h}>\hat{s}_{f}>\hat{s}^{*}$, see Figure 2.

Corollary. (to Proposition 1) Suppose that $L=0$. For any stable interior equilibrium, for a small increase in $L$ of $\epsilon>0$ low males still always accept, i.e. their ceiling is $\hat{s}_{\ell}=\bar{s}$, and the equilibrium cutoffs for high females and high males increase; hence there are fewer marriages and there is lower net welfare. Thus, the small increase in $L$ makes both types of both genders worse off, even without including the direct LoF cost.

The final result is easily derived. Because high types' cutoffs increase, low types' (who always accept) expected payoffs when encounter high types are reduced. Holding their own threshold constant, high types are always made worse off when high types on the other side raise their threshold, by a simple revealed preferences argument. ${ }^{25}$

\section{Which side is affected more?}

Consider: Is the side that bears the loss of face, here the male side, more affected than the other side? Note first that male $\ell$ types are always at least as selective as female $\ell$ types, as the lattervalways accept. For high types, the possibility of losing face may make males more reluctant to accept than females. On the other hand, this effect will increase the females' acceptance curse: it will decrease the probability that, given a female is accepted, her match

\footnotetext{
${ }^{24}$ The equilibrium tradeoff between screening and the acceptance curse was present without LoF. However, in the ARE, LoF makes playing accept less attractive for males, and this effect is stronger the more that females reject, hence a steeper reaction function.

${ }^{25}$ Consider a high type's 'higher threshold' play and payoff after the increase in $L$. If high types on the other side were to become less selective, his utility would increase, holding his threshold constant. He would also choose to raise his threshold, further increasing utility. Thus, his payoffs, even without including LoF, must be lower after the increase in $L$.
} 
was a high-type male; thus this will make high-type females more cautious. The following lemma establishes that the first effect dominates. ${ }^{26}$

Lemma 3. In any equilibrium in an ARE with Loss of Face male $h$ types are more selective than female $h$ types, i.e., $\hat{s}_{f} \leq \hat{s}_{h}$, and strictly so if the equilibrium is not a coordination failure, i.e., if $\hat{s}_{h}<\bar{s}$.

Proof. Let $L>0$. Suppose that $\hat{s}_{f} \geq \hat{s}_{h}$. Then the monotone likelihood property and equations $(18,15$ and 16) imply that

$$
F_{h}\left(\hat{s}_{\ell}\right) \geq \frac{1-F_{h}\left(\hat{s}_{h}\right)}{1-F_{h}\left(\hat{s}_{m}\right)(1+L /((1-\delta) \tilde{h}))}>1,
$$

a contradiction.

In other words, considering high types of both genders, unless LoF induces a coordination failure, the gender that is vulnerable to LoF will be more "snobbish" than the gender that is sheltered from it.

This has an interesting extension. When $L$ is small enough such that $\hat{s}_{\ell}=\bar{s}$, the probability that a high male marries "below his station": $1-F_{\ell}\left(\hat{s}_{h}^{*}\right)$, is less than $1-F_{\ell}\left(\hat{s}_{m}^{*}\right)$, the probability that a high female does so. Thus, in any stable equilibrium in an ARE with a small amount of LoF: (i) high males marry less often than high females but get better spouses on average, and (ii) low males marry more often than low females and get better spouses on average. thus for low types, a small amount of LoF on one side reduces the material (non-LoF) payoffs on the other side more.

Furthermore, only the market side that proposes may incur loss of face, suggesting a contrast from Gale and Shapley (1962). ${ }^{27}$

\subsection{Coordination failure}

Again there is the possibility of an equilibrium where all $h$ types reject with certainty. However, unlike in section 2.5, with male loss of face $L>0$, under the ARE this equilibrium is always stable. The reason is that with loss of face male $h$ types strictly prefer to reject with certainty whenever female $h$ types reject with sufficiently high probability. The intuition is straightforward: in the C-F equilibrium, high males will not respond to a tremble (nor a reduction in the cutpoint) where a small $\epsilon$ share of females play accept. Even if they are almost-surely facing a high type female, the $(1-\epsilon)$ chance of losing face outweighs the $\epsilon$ chance of marrying high.

Lemma 4. Again, there is a C-F equilibrium where $h$ types on both sides play "reject with probability 1." When one (or more) side is vulnerable to LoF this is stable (and trembling hand perfect). In this $C$-F equilibrium male $\ell$ types accept if they observe $s \leq \hat{s}_{\ell}$, where $\bar{s}>\hat{s}_{\ell}>\underline{s}$.

\footnotetext{
${ }^{26}$ Proposition 1 already found that, in a stable interior equilibrium, the market side that incurs a small loss of face becomes relatively more selective, $\hat{s}_{m}>\hat{s}_{f}>\hat{s}^{*}$. This lemma ensures that this holds at least weakly in any stable equilibrium.

${ }^{27}$ In Gale and Shapley (1962) the proposers in their deferred-acceptance algorithm are able to secure better matches in equilibrium. For instance, if the males propose to the women the men-optimal matching outcome will attain. This method is often used in practice, for instance the student-optimal algorithm in school choice. Our setup suggests a potential behavioral cost to students if LoF is relevant in this domain, unless a CAE is used.
} 
Indeed there is a possibility of coordination failure arising as one moves from the benchmark setting, where loss of face will never occur, to the ARE with a positive loss of face term. ${ }^{28}$ While for the CAE or for $L=0$ an interior equilibrium is reached whenever condition 7 holds (the coordination failure equilibrium is not stable), the coordination failure is always stable for positive $L$. In fact, the coordination failure is the unique equilibrium for a large enough increase in $L$; this follows immediately from condition (14). The following corollary compares the coordination failure to the benchmark outcome without loss of face; details are in the appendix.

Corollary. (to lemma 4) Compared to an interior equilibrium allocation of the benchmark model without loss of face, the coordination failure equilibrium in the ARE with loss of face induces

1. a lower overall marriage rate,

2. lower aggregate surplus (even without directly including loss of face in the surplus calculation),

3. and lower expected surplus (again, even without subtracting the LoF) for both types of both genders.

\subsection{Summation}

This section has demonstrated that the presence of loss of face can worsen outcomes. Where condition 7 holds, our comparative statics results (Proposition 1) demonstrate that LoF leads to fewer marriages and thus lower payoffs in an interior equilibrium. Here, LoF also makes the C-F equilibrium stable, and thus may lead to an even worse outcome, where high types never marry. If the aforementioned condition fails to hold then the C-F equilibrium may be the only stable equilibrium with or without LoF; LoF still would impose a cost (on low types), but only a direct one, with no impact on marriage outcomes.

This has a practical implication for the real-world: even if LoF is present, the equilibrium in the benchmark setup can be reached by adequate design of the matching game. Consider a case of severe loss of face, that is, $L>0$ sufficiently great, such that coordination failure arises as the only equilibrium in an ARE, and low males set a cutoff ceiling. ${ }^{29}$ Changing the setup to a CAE, where females only observe whether a marriage occurs, but not the male's action, $A$ or $R$, means that now high types set an interior cutoff, and low types always accept in any stable equilibrium. Note that if only males are vulnerable to LoF and females are not $\left(L_{m}>0\right.$ but $L_{f}=0$ ), it would be sufficient to reverse the order and let the females choose first. This may be helpful as the CAE will typically require a trustworthy intermediary, man or machine, while reversing the order would not.

\footnotetext{
${ }^{28}$ This would also hold for a Full Revelation Environment (FRE), where both sides are vulnerable to LoF; details by request.

${ }^{29}$ Note that for $L$ extremely large, the male $\ell$ types' ceiling approaches $\underline{s}$, and the rate of marriage of $\ell$ types tends to zero as well.
} 


\section{Conclusion}

\subsection{Implications}

Our work has potential normative implications. Some mechanisms and policies may be more efficient than others in the presence of LoF concerns, and firms and policymakers should take this into account. Although setting up conditionally anonymous environments may take some administrative effort, and may require a third-party monitor, we suspect that there are many cases in which it will lead to more and better matches and improve outcomes. Consider, for example, the matching of advisors and students in a Ph.D. program. A "tick box system" might work, but we imagine that some might be reluctant to participate in such an impersonal and rigid system. More generally, the use of a knowledgable, reliable, and discrete intermediary, might be more effective. Our paper motivates the use of such "matchmakers" in many contexts. ${ }^{30}$

The internet offers important tools for this. In the USA over a recent ten-year period, $17 \%$ of heterosexual couples met online (Rosenfeld and Thomas, 2012). Internet dating can minimize the LoF that comes with face-to-face transactions, allowing people to access a network of potential partners who they are not likely to run into again at the office or on the street. However, going online may not eliminate the LoF (Hitsch et al., 2010). Several sites and applications have introduced the CAE environment in some format; most notably Tinder. However, there is a trade-off: with thousands of members, each member may only view a fraction of eligible dates, and if A expresses anonymous interest B may never see A's profile. ${ }^{31}$

Preserving face is critical in resolving personal and political disagreements. Neither side may want to make a peace overture unilaterally - this can be seen as evidence of admission of guilt or weakness, and may be psychologically painful in itself. Again, where a double-blind mechanism is available, it can resolve this dilemma; if not, our model offers insight into why negotiations often fail. Often, peace talks are made in secret, and only announced if a successful agreement has been reached. Prominent diplomats and scholars have argued that privacy and secrecy are critical to successful international negotiations (Eban, 1983; Armstrong, 1993; Jonsson and Aggestam, 2008).

LoF concerns are present on both sides of the job market. Within an employment relationship, LoF might affect decisions over how much effort and care to put in and whether to apply for promotions. In Akerlof and Kranton's model (2005) "when an employee has [insider] identity, she loses some utility insofar as she deviates from the ideal action of [the firm]," and for an "outsider" this is reversed. Suppose this derives from her publicly revealed actions. Here, the firm chooses whether to pay a cost to "approve" of the employee and make her feel like an insider, and the employee simultaneously chooses whether to put in extra effort in the firm's interest. If the firm chooses not to pay this cost, i.e., to "reject" the employee, this will hurt the employee more if she has put in high effort. Thus, employees who fear such an "identity loss" or analogously, feel LoF from having publicly accepted the firm only to be rejected may only try extra-hard on tasks that they can work on in relative obscurity. The same authors' (2000) model of social exclusion can seen in terms of LoF. If being seen "acting white" involves sacrificing

\footnotetext{
${ }^{30}$ In some cases, merely encouraging theace meetings may allow colleagues to reveal their potential interest slowly and conditionally, lessening the risk of a severe LoF from a "desperate bid". This may help explain Boudreau et al (2012); who found that an exogenously assigned brief face-to-face interaction between local scientists led to significant increases in their probability of collaborating.

${ }^{31}$ Online dating may serve as a modern analogue to the traditional "matchmaker" in preserving conditional anonymity. However, the internet may expand the "gossip network" heightening reputation concerns.
} 
Black identity, a Black person may choose not to attempt admission to the dominant culture because she is uncertain whether she will be accepted by a school, employer, or White social group. On the other hand, if she can make this attempt anonymously, she can avoid the risk of a public threat to her identity, and also avoid the potential material costs of social exclusion. In fact, race-based rejection sensitivity has been found to negatively correlate with measures of African-American students' success at predominantly White universities (Mendoza-Denton et al., 2002). This concern might help justify outreach programs for underrepresented minorities; in effect "asking them first" or letting them know when they will have a high probability of succeeding.

The employer too may be vulnerable to LoF. Cawley (2003), in his guide for economists on the junior job market, writes that he has "heard faculty darkly muttering about job candidates from years ago who led them on for a month before turning them down" (pp. 49). This aggravation may involve LoF in addition to the loss of time and opportunity costs. This LoF is recognized by professional recruiters as well: "recruiters lose face when candidates pull out of accepted engagements at the last minute."32 Here too, an intermediary allowing conditional anonymity may improve outcomes. For example, the AEA signalling mechanism (Coles et al., 2010) could be extended to allow both candidates and employers to send (a limited number of) signals, and reveal only mutual matches. Both parties would then have a reasonable expectation that their investments - in the time and expense of interviewing and seminars - would pay off, and the risk of rejection and loss of reputation would be lessened. ${ }^{33}$

\subsection{Suggestions for future work}

This modeling in our paper can be expanded in several ways, relaxing many of our assumptions and generalizing our results. In a model allowing both inherent LoF and reputation, the effects of revealing offers on match efficiency may be complex. If a player is known to be vulnerable to LoF, his making an offer might actually be interpreted as a signal of his confidence that he will be accepted, thus a positive signal about his own type. Whenever a player rejects another, there is some possibility that he did so merely to avoid losing face; noting this possibility should presumably soften the blow to a player's reputation when he is rejected.

Relaxing the assumptions further, preferences over types may be heterogeneous, or may involve a horizontal component, this may also change the equilibrium reputation effects of revealing offers. It also may be interesting to consider the effects of a player who is either altruistic, suffering when the other player loses face, or spiteful, relishing in making others lose face. Consider a sequential game where only the first mover is vulnerable to LoF and the second mover is a known altruist. Here the first-mover might manipulate this altruism, playing "accept" and in effect guilting the second-mover into matching with her; this could lead to inefficient matching.

Empirically, our anecdotal and referential evidence for LoF should be supplemented by experimental evidence. Field experiments (or contextual lab work) in the mold of Lee et al. (2011) will help identify preferences and beliefs. Abstract "induced values" experiments may also shed light on strategic play and coordination in our simple environment. ${ }^{34}$ As well as strengthen-

\footnotetext{
${ }^{32}$ Leslie Merrow, Staffing Consultancy Principal, "How to Avoid the Counter-Offer Trap," Friday, August 17, 2007. <http://directsearchalliance.blogspot.com/2007/08/how-to-avoid-counter-offer-trap.html>

${ }^{33}$ For senior administrative academic positions "airport interviews" are also used "to interview a large number of candidates...with a degree of confidentiality." These occur at a local airport hotel rather than on-campus, perhaps to minimize reputational consequences on both sides (Williamson, 2008).

${ }^{34}$ These papers involve symmetric horizontal matching preferences in an anonymous laboratory setting.
} 
ing the evidence for the existence of the LoF motivation, these experiments should examine the causes and correlates of LoF, and its efficiency consequences in various environments. Do people act strategically to minimize their own risk of LoF? Will they be willing to pay to preserve the anonymity of their offers? Who is most affected by loss of face and when ${ }^{35}$ How can these issues be addressed to improve matching efficiency in real-world environments? We hope that future research will offer empirical answers to these questions.

\section{References}

Adachi, H. (2003). A search model of two-sided matching under nontransferable utility. Journal of Economic Theory 113(2), 182-198.

Akerlof, G. A. and R. E. Kranton (2005). Identity and the Economics of Organizations. Journal of Economic Perspectives 19(1), 9-32.

Akerlof, George A. and Kranton, Rachel E. (2000, aug). Economics and Identity. The Quarterly Journal of Economics 115(3), 715-753.

Albrecht, J., P. A. Gautier, and S. Vroman (2006). Equilibrium directed search with multiple applications. Review of Economic Studies 73(4), 869-891.

Anderson, A. and L. Smith (2010). Dynamic matching and evolving reputations. The Review of Economic Studies 77(1), 3-29.

Armstrong, T. (1993). Breaking the ice: rapprochement between East and West Germany, the United States and China, and Israel and Egypt. United States Inst of Peace Press.

Battigalli, P. and M. Dufwenberg (2009). Dynamic psychological games. Journal of Economic Theory.

Baxter, L. A. and W. W. Wilmot (1984). Secret tests. Human Communication Research 11(2), $171-201$.

Becker, G. S. (1973). A Theory of Marriage: Part I. Journal of Political Economy 81(4), 813.

Bernheim, B. D. (1994). A Theory of Conformity. Journal of Political Economy 102(5), 841.

Bonita London, Geraldine Downey, Cheryl Bonica, Iris Paltin (2007). Social Causes and Consequences of Rejection Sensitivity. Journal of Research on Adolescence 17(3), 481-506.

Echenique and Yariv (2011) and Pais et al. (2012) allow subjects to make and reject/accept offers, one at a time for a certain duration, in small groups. Echenique and Yariv (2011) offer evidence that stability is a good predictor of market outcomes with complete information over preferences. Pais et al. (2012) find that making it costly to send offers (akin to a standard search friction) leads, unsurprisingly, to fewer and slightly less ambitious offers, and to less efficiency and less stable matchings. Incomplete information boosts both stability and efficiency. Haruvy and Utku Ünver (2007) use a more rigid matching environment and find that proposer-side optimal stable matches are reached in the majority of cases regardless of the information structure. Other related papers include Kagel and Roth (2000), Nalbantian and Schotter (1995), Molis and Veszteg (2010). However, none of these (i) rely on homegrown preferences and beliefs over social interactions or partnerships, (ii) have face-to-face interaction, (iii) test the single-shot matching of our model, (iii) compare environments such as our CAE and ARE, nor (iv) have a subjects' previous choices and history reported to later matches.

${ }^{35}$ The psychological evidence cited in the introduction points to heterogeneity in LoF: subjects with observable (e.g., sex, race) and unobservable (e.g., popularity) differences may be particularly concerned with LoF. 
Boudreau, K., Ganguli I, P. Gaule, E. Guinan, and K. Lakhani (2012). Colocation and Scientific Collaboration: Evidence from a Field Experimentsns. Working paper. Harvard Business School Electronic.

Burdett, K. and M. Coles (2006). Marriage and class. Journal of Economic Theory 129, 81-113.

Burdett, K. and M. G. Coles (1999). Long-Term Partnership Formation: Marriage and Employment. The Economic Journal 109(456), 307-334.

Cawley, J. (2003). A Guide (and advice) for economists on the U.S. junior academic job market. Job Openings for Economists.

Chade, H. (2006). Matching with noise and the acceptance curse. Journal of Economic Theory 129(1), 81-113.

CNN International (2005). Speed dating's executive makeover (http://edition.cnn.com/2005/BUSINESS/01/27/speed.networking/index.html).

Coles, P., J. Cawley, P. B. Levine, M. Niederle, A. E. Roth, and J. J. Siegfried (2010). The job market for new economists: A market design perspective. The Journal of Economic Perspectives 24 (4), 187-206.

Collins, N. and J. Goyder (2013). Speed Dating: a process of forming undergraduate student groups. eCULTURE 1(1), 9.

Dixit, A. (1986). Comparative statics for oligopoly. International Economic Review 27(1), $107-122$.

Douglas, W. (1987). Affinity-testing in initial interactions. Journal of Social and Personal Relationships 4(1), 3-15.

Eastwick, P. W. and E. J. Finkel (2008). Speed-dating: A powerful and flexible paradigm for studying romantic relationship initiation. Handbook of relationship initiation, 217-234.

Eban, A. S. (1983). The New Diplomacy: International Affairs in the Modern Age. Random House NY.

Echenique, F. and L. Yariv (2011). An experimental study of decentralized matching. Mimeo.

Eisenberger, N. I. and M. D. Lieberman (2004). Why rejection hurts: a common neural alarm system for physical and social pain. Trends in Cognitive Sciences 8(7), 294-300.

Falk, A. and U. Fischbacher (2006, feb). A theory of reciprocity. Games and Economic Behavior $54(2), 293-315$.

Gale, D. and L. S. Shapley (1962, jan). College Admissions and the Stability of Marriage. The American Mathematical Monthly 69(1), 9-15.

Geery, B. J. (1996). Contests Combat Call Reluctance (Sidebar). Telemarketing and Call Center Solutions 15, 112-115.

Goffman, E. (2005). Interaction Ritual: Essays in Face to Face Behavior. Aldine Transaction. 
Hahn, F. H. (1962). The stability of the Cournot oligopoly solution. The Review of Economic Studies, 329-331.

Haruvy, E. and M. Utku Ünver (2007). Equilibrium selection and the role of information in repeated matching markets. Economics Letters 94(2), 284-289.

Hitsch, G. J., A. Hortacsu, and D. Ariely (2010). Matching and Sorting in Online Dating. The American Economic Review 100(1), 130-163.

Hugh-Jones, D. and D. Reinstein (2010). Losing face. University of Essex, Department of Economics, Economics Discussion Papers 691.

Jonsson, C. and K. Aggestam (2008). Diplomacy and Conflict Resolution. The SAGE Handbook of Conflict Resolution, 33 .

Kagel, J. H. and A. E. Roth (2000). The dynamics of reorganization in matching markets: A laboratory experiment motivated by a natural experiment. The Quarterly Journal of Economics 115(1), 201-235.

Koszegi, B. (2006). Ego Utility, Overconfidence, and Task Choice. Journal of the European Economic Association 4(4), 673-707.

Lee, S., M. Niederle, H. R. Kim, and W. K. Kim (2011). Propose with a rose? signaling in internet dating markets. Technical report, National Bureau of Economic Research.

Mendoza-Denton, R., G. Downey, V. J. Purdie, A. Davis, and J. Pietrzak (2002). Sensitivity to status-based rejection: implications for African American students' college experience. Journal of personality and social psychology 83(4), 896.

Milgrom, P. and C. Shannon (1994). Monotone comparative statics. Econometrica: Journal of the Econometric Society, 157-180.

Molis, E. and R. F. Veszteg (2010). Experimental results on the roommate problem. CORE DP 11.

Nalbantian, H. R. and A. Schotter (1995). Matching and efficiency in the baseball free-agent system: an experimental examination. Journal of Labor Economics, 1-31.

Pais, J., A. Pinter, and R. F. Veszteg (2012). Decentralized matching markets: a laboratory experiment. Mimeo: ISEG-Departamento de Economia.

Putnam, R. D. (1995). Bowling alone: America's declining social capital. Journal of democracy $6(1), 65-78$.

Rabin, M. (1993). Incorporating Fairness into Game Theory and Economics. American Economic Review 83(5), 1281-1302.

Rosenfeld, M. J. and R. J. Thomas (2012). Searching for a Mate: The Rise of the Internet as a Social Intermediary. American Sociological Review 7r(4), 523-527.

Selten, R. (1975). Reexamination of the perfectness concept for equilibrium points in extensive games. International journal of game theory 4 (1), 25-55. 
Shanteau, J. and G. F. Nagy (1979). Probability of acceptance in dating choice. Journal of Personality and Social Psychology 37(4), 522.

Vives, X. (2005). Complementarities and games: New developments. Journal of Economic Literature, 437-479.

Williams, K. D. (2007). Ostracism. Annu Rev Psychol 58, 425-452.

Williamson, D. (2008). Whiffing the Airport Interview - The Chronicle of Higher Education.

\section{A Terminal information sets and game trees}

The set of end nodes of the game, defined by their histories, is $h=[h 1, h 2, h 3, h 4]=[A A, A R, R A, R R]{ }^{36}$ Let $\bar{I}_{f}$ be the collection of $f$ 's terminal information sets over these endnodes, and $\bar{I}_{m}$ be $m$ 's information partition. Since neither player "has the move" at the terminal node, we give each history two boxes to depict each player's terminal information set; $h_{j}(m)$ and $h_{j}(f)$ are the same (for $j \in\{1,2,3,4\}$ ).

Two versions of this game are illustrated in the trees below, which display only material payoffs, followed by a specification of the terminal information partitions for each case.

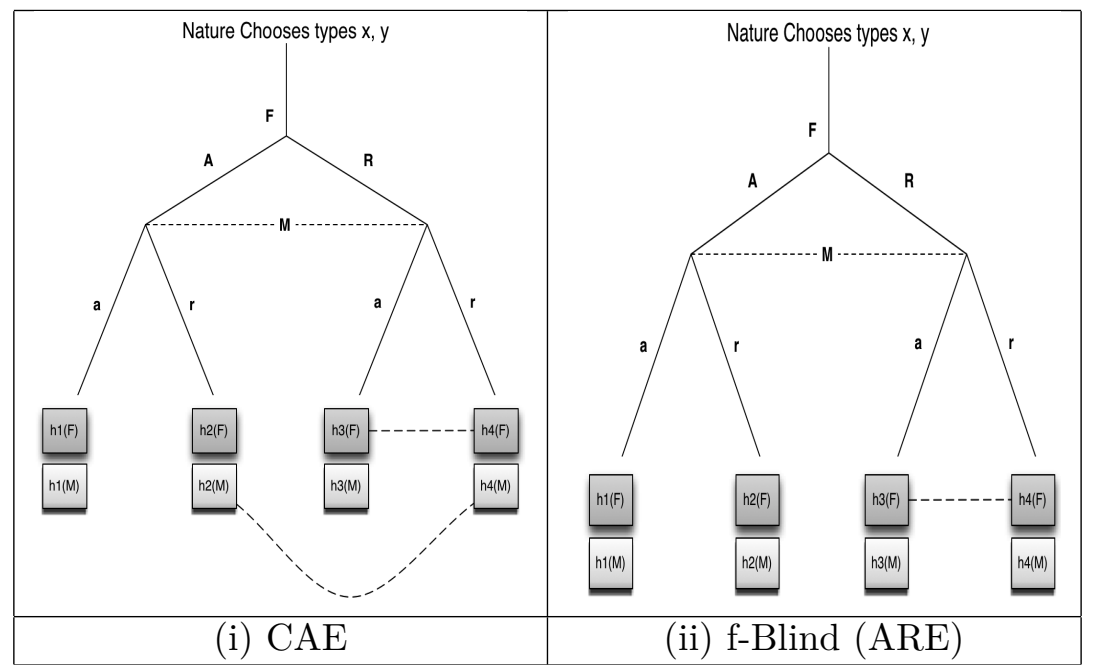

Figure 3: Terminal Information structures

(i) Conditionally Anonymous (CAE):

$\bar{I}_{F}=(\{\langle A, A\rangle\},\{\langle A, R\rangle\},\{\langle R, A\rangle,\langle R, R\rangle\})$ and $\bar{I}_{m}=(\{\langle A, A\rangle\},\{\langle A, R\rangle\langle R, R\rangle\},\{\langle R, A\rangle\})$.

(ii) $\mathrm{f}$ is "blind":

$\bar{I}_{f}=(\{\langle A, A\rangle\},\{\langle A, R\rangle\},\{\langle R, A\rangle,\langle R, R\rangle\}) ; \bar{I}_{M}=(\{\langle A, A\rangle\},\{\langle A, R\rangle\},\{\langle R, A\rangle\},\{\langle R, R\rangle\})$.

Note, for example that in the simultaneous CAE the $\{A, A\}$ terminal information set is a singleton for both players, while the histories where a player played "reject" are part of the same terminal information set (for that player).

\footnotetext{
${ }^{36}$ We leave nature's move out of these histories; it does not affect our discussion. For completeness we can assume that players never learn the other players' types. Thus, in our model LoF will only depend on the conditional expectation of the other player's type, not the type itself.
} 


\section{B Equivalence of sequential and simultaneous environments; CAE equivalence to no-LoF}

\section{i. Simultaneous depiction}

Under the CAE both $\mathrm{m}$ and $\mathrm{f}$ choose simultaneously, and the terminal information set only distinguishes (A,A) from all other outcomes. This is automatically observed if we assume material/marriage payoffs are observed. LoF is thus avoided.

Under the ARE both $\mathrm{m}$ and $\mathrm{f}$ choose simultaneously. Here the male has three terminal information sets, $\{\langle A, A\rangle\},\{\langle A, R\rangle\}$ and $\{\langle R, A\rangle,\langle R, R\rangle\}$, while the female has all four terminal information sets. Thus, by our definition, the male can incur LoF but the female can not. This is, of course, also strategically equivalent to setting the female $L$ term to zero.

\section{ii. Sequential depiction}

CAE: $m$ chooses first and then $f$, but the first mover's choice is not observed by the second mover when she chooses (so the order of choice obviously doesn't matter.) Strategically and payoff-wise, this is equivalent to a simultaneous choice. The terminal information set only distinguishes AA from all other outcomes (again this is automatic if we assume material payoffs are observed). LoF is thus avoided. This is thus strategically and payoff-equivalent to the simultaneous CAE.

ARE: $m$ chooses first and then f observes m's choice, and chooses. Both parties learn (i.e., have a distinct terminal information set) if there is a marriage (A,A). Here, whether or not the terminal information set also distinguishes $(\mathrm{R}, \mathrm{A})$ from $(\mathrm{R}, \mathrm{R})$ is inconsequential, because $\mathrm{f}$ will never choose $\mathrm{A}$ if $\mathrm{m}$ chose $\mathrm{R}$. Thus the male may incur LoF but the female never will. Ruling out the females dominated strategies, this is thus strategically and payoff equivalent to the simultaneous FRE.

\section{Proofs}

\section{C.1 Exhaustive proof that players without LoF use threshold strategies in settings with and without LoF}

Define $q\left(x, x^{\prime}\right)$ as the probability that a player of type $x$ accepts a match of type $x^{\prime}$. Writing this in terms of likelihood ratios, the acceptance condition becomes $\frac{p f_{h}(s) q(h, h) \tilde{h}+(1-p) f_{\ell}(s) q(\ell, h) \tilde{\ell}}{p f_{h}(s) q(h, h)+(1-p) f_{\ell}\left(s_{f}\right) q(\ell, h)} \geq \delta \tilde{h}$, which simplifies to

$$
\frac{f_{h}(s)}{f_{\ell}(s)} p q(h, h)(\tilde{h}-\delta \tilde{h}) \geq(1-p) q(\ell, h)(\delta \tilde{h}-\tilde{\ell}) .
$$

Using the monotone likelihood ratio property and our other assumptions over the density we know the ratio $\frac{f_{h}\left(s_{f}\right)}{f_{\ell}\left(s_{f}\right)}$ increases from 0 to 1 over the range of $s$. The right hand side is positive as long as $\ell$ 's accept with at least some positive probability. We can rule out an equilibrium where $\ell$ 's always reject even when $\ell$ 's suffer from LoF. Regardless of $h$ 's behavior, since $\ell$ 's will always reciprocate an acceptance, if an $\ell$ observes a low enough signal the expected benefit of marrying an $\ell$ will outweigh the expected LoF from being rejected by an $h$. Ruling this out implies that $h$ 's must reject when they observe the lowest signals, as these almost surely come from $\ell$ 's. Thus $h$ 's must have a cutoff best response $\hat{s}>\underline{\mathbf{s}}$. 


\section{C.2 Proof that best responses are the same if LoF is a continuous function of second-order beliefs}

\section{Claim: Optimal strategies are the same whether}

- Discrete-LoF: LoF is a positive constant when she knows for sure that he played accept and she plays reject and zero otherwise or

- Continuous-LoF: LoF increases in the others' assessment of the probability he played accept when she plays reject, with a maximum value of $L$ if this probability is 1 (and $i$ still incurs no LoF if $j$ plays accept).

... for all types and genders in both ARE and CAE environments.

\section{Case-by-case proof}

CAE (same for both genders) With discrete-LoF, there will be no LoF for anyone, hence this does not depend on actions. With continuous LoF, $i$ 's LoF depends on $j$ 's assessment of $i$ "s action, which does not depend on $i$ 's actual action (because it is unobserved). Because LoF is additively separable in the utility function, best response functions are the same as with discrete LoF.

ARE - males (first-movers) With discrete or continuous-LoF, Males's actions are always observed. If the female rejects, then if the male accepts he gets LoF $L$ in the discrete case, and the same maximal LoF $L$ (based on assessed probability 1 ) in the continuous case. If either the male rejects or the female accepts, he gets no LoF in either environment. Thus the optimal strategies will be the same with discrete or continuous LoF, assuming females play the same strategies in each of these environments (as we show below).

ARE - females (second movers) With discrete LoF, females never suffer LoF (because they will never accept if the male rejects). With continuous LoF, if a male rejects, he knows the female will reject (i.e., his assessment puts probability 1 on her playing reject), so she gets no LoF. Thus the female never suffers LoF, as in the Discrete case, thus her best response function is unchanged.

\section{C.3 Proof of Lemma 2}

The use of thresholds $\hat{s}_{h}, \hat{s}_{\ell} \in[\underline{s}, \bar{s}]$ follows from condition (12) and the monotone likelihood property, see text. Since $\delta \tilde{h}>\tilde{\ell}>\delta \tilde{\ell}$ by assumption, male $h$ types accept if $s_{f} \geq \hat{s}_{h}$ and male $\ell$ types accept if $s_{f} \leq \hat{s}_{\ell}$. $\hat{s}_{h}>\underline{s}$ and $\hat{s}_{\ell}>\underline{s}$, since $\delta \tilde{h}>\tilde{\ell}>(1-\delta) \tilde{\ell}$ and for $s_{f}=\underline{s}$ the probability that the female match is of type $\ell$ is 1 . Noting that condition (13) implies condition (12) for $\tilde{x}=\tilde{\ell}$ and that condition (14) implies that (12) does not hold for $\tilde{x}=\tilde{h}$ establishes the lemma.

\section{C.4 Proof of Proposition 1}

The changes in $\hat{s}_{f}$ and $\hat{s}_{h}$ from $\hat{s}$ associated with the $\epsilon$ increase in $L$ are determined by the system of equations (18) and (15). The total differential of (18) for $L=\epsilon$ small enough for the 
ceiling $\hat{s}_{\ell}^{*}=\bar{s}$ to hold is

$$
\frac{\partial \frac{f_{h}\left(\hat{s}_{f}\right)}{f_{\ell}\left(\hat{s}_{f}\right)}}{\partial s} \frac{p}{1-p} \frac{(1-\delta) \tilde{h}}{\delta \tilde{h}-\tilde{\ell}} \Delta \hat{s}_{f}=\frac{f_{h}\left(\hat{s}_{h}\right)}{\left(1-F_{h}\left(\hat{s}_{h}\right)\right)^{2}} \Delta \hat{s}_{h}
$$

where the $\Delta$ terms denote the changes in $\hat{s}_{f}$ and $\hat{s}_{h}$. Note that this already implies that the equilibrium cutoffs for high females and males move in the same direction as long as $L$ is sufficiently small to ensure that the ceiling $\hat{s}_{\ell}=\bar{s}$. For marginal changes we have

$$
\frac{\partial \hat{s}_{f}}{\partial \hat{s}_{h}}=\frac{\frac{f_{h}\left(\hat{s}_{h}\right)}{\left(1-F_{h}\left(\hat{s}_{h}\right)\right)^{2}}}{\frac{\partial \frac{f_{h}\left(\hat{s}_{f}\right)}{f_{\ell}\left(\hat{s}_{f}\right)}}{\partial s} \frac{p}{1-p} \frac{(1-\delta) \tilde{h}}{\delta \tilde{h}-\tilde{\ell}}}>0
$$

Conducting a similar exercise for (15) yields

$$
\frac{\partial \frac{f_{h}\left(\hat{s}_{h}\right)}{f_{\ell}\left(\hat{s}_{h}\right)}}{\partial s} \frac{p}{1-p} \frac{(1-\delta) \tilde{h}}{\delta \tilde{h}-\tilde{\ell}} \Delta \hat{s}_{h}=\frac{f_{h}\left(\hat{s}_{f}\right)(L+(1-\delta) \tilde{h}) \Delta \hat{s}_{f}+F_{h}\left(\hat{s}_{f}\right) \delta \tilde{l}}{(1-\delta) \tilde{h}\left(1-F_{h}\left(\hat{s}_{f}\right)(1+L /((1-\delta) \tilde{h}))\right)^{2}}
$$

Plugging in $\Delta \hat{s}_{f}$ from above and focusing on the neighborhood of $L=0$, where $\hat{s}_{f}=\hat{s}_{h}=\hat{s}^{*}$, yields that for marginal variations in $L$

$$
\frac{\partial \hat{s}_{f}}{\partial L}=\frac{f_{h}\left(\hat{s}^{*}\right) \frac{F_{h}\left(\hat{s}^{*}\right)}{(1-\delta) \tilde{h}}}{\left(1-F_{h}\left(\hat{s}^{*}\right)\right)^{4}\left(\frac{\partial \frac{f_{h}\left(\hat{s}^{*}\right)}{f_{\ell}\left(\hat{s}^{*}\right)}}{\partial s}\right)^{2}\left(\frac{p}{1-p} \frac{(1-\delta) \tilde{h}}{\delta \tilde{h}-\tilde{\ell}}\right)^{2}-f_{h}\left(\hat{s}^{*}\right)^{2}}
$$

That is, a marginal increase of $L$ at $L=0$ yields a (weak) increase of $\hat{s}_{f}$ (and $\hat{s}_{h}$ by (21)) if, and only if

$$
\frac{\partial \hat{s}_{f}}{\partial \hat{s}_{h}}=\frac{f_{h}\left(\hat{s}^{*}\right)}{\left(1-F_{h}\left(\hat{s}^{*}\right)\right)^{2}} \frac{p}{1-p} \frac{(1-\delta) \tilde{h}}{\delta \tilde{h}-\tilde{\ell}} \frac{\partial \frac{f_{h}\left(\hat{s}^{*}\right)}{f_{\ell}\left(\hat{s}^{*}\right)}}{\partial s}<1 .
$$

\section{C.5 Proof of Lemma 4}

Trembling hand perfection requires the equilibria of a sequence of games with $\epsilon$ trembles to converge to the equilibrium of the game without trembles as $\epsilon$ approaches zero. In a game perturbed by an $\epsilon$ tremble agents play mixed strategies that place at least probability $\epsilon$ on each pure strategy. In the unperturbed game with $L>0$ males strictly prefer to reject with certainty if female $h$ types do so as well. Hence, also for small perturbations resulting in $q_{m}^{\epsilon}(h, h)>0$ (but still small) males strictly prefer to reject with certainty. Hence, male $h$ type's best response converges to its counterpart in the equilibrium in the unperturbed game as $\epsilon$ converges to 0 . This implies that also female $h$ types' best reply converges to its counterpart in the equilibrium in the unperturbed game. Hence, the equilibrium is indeed trembling hand perfect.

The last statement in the proposition follows from the fact that $\hat{s}_{m}=\bar{s}$, which implies that condition (13) cannot hold, thus Lemma 2 must apply.

The proof for best-reply stability follows immediately from the above.

\section{C.6 Details for corollary to lemma 4}

(i) In the benchmark case all $\ell$ accept and some $h$ do. In the C-F equilibrium with LoF no $h$ accept, but all male $\ell$ accept. Suppose also the "best case" that all female $\ell$ 's also accept. (As 
noted, this is counterfactual; female $\ell$ 's will reject against the highest signals). This yields the reduced marriage rates given in the corollary.

(ii) The reduction in aggregate payoffs follows directly from the linear marriage production function.

(iii) The argument for the reduced expected surplus of each gender/type relies on a revealed preference argument. Consider going in the opposite direction, from the C-F equilibrium to the benchmark. The strict increase in the rate of acceptance for $h$ 's requires that $h$ 's must be better off when they accept, as their outside option remains the same, and are thus better off in expectation. (An analogous argument holds for the increase in the acceptance rate for female $\ell$ 's shown later). Furthermore, when playing $A$, both $\ell$ 's and $h$ 's are better off when $h$ 's are in the market; their expected mate will be of higher quality.

As argued earlier, even without a primal loss of face, being observed playing accept may have a negative reputational consequence (which itself may be painful). In a multi-period interaction this may also lead to material losses, including worse matching prospects in later periods. We demonstrate this below in a two-period model. We compare the case where a player's choice in the first period is private, to a case where it is revealed to her second-period match. We show that in the latter case playing accept in the first period worsens one's continuation value, making first-period incentives equivalent to our primal LoF model in Section 3.

\section{C.7 A Repeated Matching Market}

Basic payoffs and the information structure follows from section 2.5. However, we now assume that the market remains open for two periods. We give the timing below; note that stages 1-3 are essentially the same as above.

We focus on settings that result in stable, interior equilibria, i.e., settings where Condition 7 holds.

\section{C.8 Timing}

1. Individuals in $M$ and $F$ are matched to each other randomly. In each match $(m, f)$ each individual $i$ obtains a noisy signal $s_{j}$ about the other one's type $x_{j}$.

2. After observing the signal individuals simultaneously decide on whether to accept or reject the match.

3. Pairs in which both individuals played accept form a marriage and are removed from the market and receive payoffs.

4. The remaining individuals are again matched into pairs $(m, f)$ randomly. Again, in each match $(m, f)$ each individual obtains a noisy signal $s_{j}$ about the other's type $x_{j} .{ }^{37}$

5. In an "Asymmetric Partial Revelation Environment" (APRE), but not the "Conditionally Anonymous Environment" (CAE), females observe their (male) match's action in the previous stage, but not vice-versa.

6. After observing the signal individuals simultaneously decide on whether to accept or reject the match.

\footnotetext{
${ }^{37}$ For brevity, we do not separately index the first and second period match, nor the first and second period signals - these will be clear from context.
} 
7. Pairs in which both individuals accepted form a marriage, all others remain single. The market closes and payoffs are realized.

\section{C.9 Conditionally anonymous environment (CAE)}

We solve backwards from the second stage; this solution is identical to that of the one-period model without LoF, re-labeling some variables; we thus give only a few key equations and results below. Denote the share of $h$ agents on each side in period 2 by $p_{2}$, which will depend on players' equilibrium strategies. From the assumption that $f_{h}(\underline{s})=0$ we know that a strictly positive measure of agents remains in the market at $t=2 .^{38}$ Thus $0<p_{2}<1$. In the second period, an agent who receives a signal $s_{j}$ assesses the probability of facing a high type as

$$
\operatorname{pr}\left(h \mid s_{j}\right)=\frac{p_{2} f_{h}\left(s_{j}\right)}{p_{2} f_{h}\left(s_{j}\right)+\left(1-p_{2}\right) f_{\ell}\left(s_{j}\right)} .
$$

\section{C.9.1 Equilibrium Behavior}

\section{CAE: Second period}

As there is no further reputation motive in the final period, nor an intrinsic LoF, nor is previous play observed in the CAE, this period is equivalent to the CAE (or benchmark model without LoF) from section 2.5, replacing $p$ with $p_{2}$, and noting that $s$ refers to the second-period signal. The results follow (restating the earlier lemma and corollary with minor adjustments to notation).

Lemma 5. [CAE Second Stage] In a CAE, if an interior stable equilibrium exists (conditions for this are as in the main text), then for the subgame starting in period 2:

1. low types always accept,

2. high types use symmetric cutoff strategies, accepting if $s>\hat{s}_{m}=\hat{s}_{f}:=\hat{s}^{*}$, and

3. $\hat{s}^{*} \in(\underline{s}, \bar{s})$.

Note $\hat{s}^{*}$ is defined by equation (5), replacing $p$ with $p_{2}$.

Corollary. Expected payoffs for types $\ell$ and $h$ in an interior stable equilibrium of the secondperiod subgame of the CAE (or for any strategy profile where low types always accept) are

$$
\begin{aligned}
& v_{2}(\ell)=\delta \tilde{\ell}+p_{2}\left(1-F_{\ell}\left(\hat{s}^{*}\right)\right)(\tilde{h}-\delta \tilde{\ell})+\left(1-p_{2}\right)(\tilde{\ell}-\delta \tilde{\ell}), \text { and } \\
& v_{2}(h)=\delta \tilde{h}+p_{2}\left(1-F_{h}\left(\hat{s}^{*}\right)\right)^{2}(\tilde{h}-\delta \tilde{h})-\left(1-p_{2}\right)\left(1-F_{\ell}\left(\hat{s}^{*}\right)\right)(\delta \tilde{h}-\tilde{\ell}) .
\end{aligned}
$$

\footnotetext{
${ }^{38}$ To violate this both types would have to play "always accept" in the first stage, expecting to remain unmarried with probability 1 conditional on reaching stage 2 . This would be an equilibrium if the expected match quality in period 1 were high enough for an $h$ type to accept regardless of the signal:$$
p \geq \frac{f_{\ell}(\underline{s})(\delta \tilde{h}-\tilde{\ell})}{f_{\ell}(\underline{s})(\delta \tilde{h}-\tilde{\ell})+f_{h}(\underline{s})(1-\delta) \tilde{h}} .
$$

This case is excluded by the assumption that $f_{h}(\underline{s})=0$. Moreover, if this assumption were not made and this were an equilibrium, it would not be stable nor trembling hand perfect, since an agent facing a signal $s=\underline{s}$ in $t=1$ would prefer to take another chance in $t=2$ if the proportion of $\ell$ and $h$ agents were to remain the same, as it would under a random tremble.
} 
Note $v_{2}(h)>v_{2}(\ell) .{ }^{39}$ The number of marriages is $\left(1-p_{2}\right)^{2}+2 p_{2}\left(1-p_{2}\right)\left(1-F_{\ell}\left(\hat{s}^{*}\right)\right)+p_{2}^{2}(1-$ $\left.F_{h}\left(\hat{s}^{*}\right)\right)^{2}$, which strictly decreases in $\hat{s}^{*}$.

\section{CAE: First Stage}

A similar reasoning applies to the first stage: an agent of type $x_{i}$ who observes a signal $s_{j}$ finds it profitable to accept if and only if the expected payoff exceeds her continuation value $v_{2}\left(x_{i}\right)$ after playing reject. As, in the $\mathrm{CAE}$, the continuation value from rejecting or being rejected are both zero, we only need to see if the payoff from an expected marriage exceeds this. Stating this in terms of gains relative to the continuation value we have:

$$
p f_{h}(s) q_{1}(x, h)\left(\tilde{h}-v_{2}(x)\right)+(1-p) f_{\ell}(s) q_{1}(x, \ell)\left(\tilde{\ell}-v_{2}(x)\right) \geq 0 .
$$

Recall, a high type prefers marrying a high type over remaining single, which she prefers over marrying a low type; thus her continuation value satisfies $\tilde{\ell}<\delta \tilde{h}<v_{2}(h)<\tilde{h}$ (the strictness of the latter inequalities follows from $0<p_{2}<1$, previously demonstrated, and from an interior equilibrium in period 2 , under conditions specified). Thus, for a high type the first additive term in inequality (24) must be positive and the second term negative. By the monotone likelihood property, as the signal $s$ increases the first term increases in magnitude and the second term decreases in magnitude (and this holds for both types). Thus the high type will set a floor threshold. By a similar argument, for a low type $(x=\ell)$ the first term must be positive but the second term may have either sign. If it is negative the low type must also set a floor threshold. If both terms are positive the low type will always accept in the first period (a trivial floor at $\underline{s})$.

Thus both types will set a floor threshold: there are values $\hat{s}_{x} \in[\underline{s}, \bar{s}]$ for $x=\ell, h$, such that the agent accepts only if $s \geq \hat{s}_{x}$. Similarly to the second stage, where interior, these are implicitly defined by

$$
p f_{h}\left(\hat{s}_{x}\right)\left(1-F_{x}\left(\hat{s}_{h}\right)\right)\left(\tilde{h}-v_{2}(x)\right)=(1-p) f_{\ell}\left(\hat{s}_{x}\right)\left(1-F_{x}\left(\hat{s}_{\ell}\right)\right)\left(v_{2}(x)-\tilde{\ell}\right),
$$

where the left side represents the expected benefit from marrying high relative to staying alone, and the right side the expected "loss" from marrying low relative to staying alone.

Lemma 6. The first period cutoffs satisfy $\hat{s}_{h}>\hat{s}_{\ell}$.

Proof: Rearranging (25) yields cutoff values $\hat{s}_{h}$ and $\hat{s}_{\ell}$ defined by

$$
\begin{aligned}
\frac{p f_{h}\left(\hat{s}_{h}\right)}{(1-p) f_{\ell}\left(\hat{s}_{h}\right)} & =\frac{v_{2}(h)-\tilde{\ell}}{\tilde{h}-v_{2}(h)} \frac{1-F_{h}\left(\hat{s}_{\ell}\right)}{1-F_{h}\left(\hat{s}_{h}\right)} \text { and } \\
\frac{p f_{h}\left(\hat{s}_{\ell}\right)}{(1-p) f_{\ell}\left(\hat{s}_{\ell}\right)} & =\frac{v_{2}(\ell)-\tilde{\ell}}{\tilde{h}-v_{2}(\ell)} \frac{1-F_{\ell}\left(\hat{s}_{\ell}\right)}{1-F_{\ell}\left(\hat{s}_{h}\right)} .
\end{aligned}
$$

Since $v_{2}(h)>v_{2}(\ell)$ as shown above, the contradiction to the lemma, $\hat{s}_{h} \leq \hat{s}_{\ell}$ implies that

$$
\frac{1-F_{h}\left(\hat{s}_{\ell}\right)}{1-F_{h}\left(\hat{s}_{h}\right)} \leq \frac{1-F_{\ell}\left(\hat{s}_{\ell}\right)}{1-F_{\ell}\left(\hat{s}_{h}\right)}
$$

\footnotetext{
${ }^{39}$ This must hold as high types could always adopt the same "always accept" strategy as low types and gain a strictly higher payoff than low types. In doing so, both high and low types would always marry when they meet a low type, but high types would be more likely to marry when meeting another high type (and high type's unmarried payoff are also higher).
} 
Rewriting this in terms of integrals yields

$$
1+\frac{\int_{\hat{s}_{h}}^{\hat{s}_{\ell}} f_{\ell}(s) d s}{\int_{\hat{s}_{\ell}}^{\bar{s}} f_{\ell}(s) d s} \leq 1+\frac{\int_{\hat{s}_{h}}^{\hat{s}_{\ell}} f_{h}(s) d s}{\int_{\hat{s}_{\ell}}^{\bar{s}} f_{h}(s) d s} .
$$

This becomes

$$
\frac{\int_{\hat{s}_{h}}^{\hat{s}_{\ell}} f_{h}(s) d s}{\int_{\hat{s}_{h}}^{\hat{s}_{\ell}} f_{\ell}(s) d s} \geq \frac{\int_{\hat{s}_{\ell}}^{\bar{s}} f_{h}(s) d s}{\int_{\hat{s}_{\ell}}^{\bar{s}} f_{\ell}(s) d s} .
$$

The monotone likelihood ratio property implies the contrary, since

$$
\frac{f_{h}(s)}{f_{\ell}(s)}<\frac{f_{h}\left(\hat{s}_{\ell}\right)}{f_{\ell}\left(\hat{s}_{\ell}\right)}<\frac{f_{h}\left(s^{\prime}\right)}{f_{\ell}\left(s^{\prime}\right)}
$$

for all $s<\hat{s}_{\ell}<s^{\prime}$.

Thus high types will be more selective than low types in stage 1 .

Equation (25) leads to several results. Recalling assumption 2, that extreme signals fully reveal types, low types will "accept always" only if $v_{2}(\ell) \leq \tilde{\ell}$. This cannot be ruled out: the low types' continuation value may be below the value of marrying low, as they may be rejected in stage 2 , which would leave them worse off.

In contrast, high types do not "accept always" - this would require $v_{2}(h) \leq \tilde{\ell}$, but as we have shown $v_{2}(h)>\tilde{h}$ and $\delta \tilde{h}>\tilde{\ell}$ by assumption. High types will also not "reject always"; this would be optimal only if $v_{2}(h) \geq \tilde{h}$, which could hold only if there are no low types in stage 2 . But we know $p_{2}<1$ : even if all $\ell$ 's accept in stage 1 some will meet high types, give off low signals, and be rejected, surviving to stage 2. Hence, $\underline{s}<\hat{s}_{h}<\bar{s}$, and $\underline{s} \leq \hat{s}_{\ell}<\hat{s}_{h}$. This fully characterizes equilibrium strategies, summarized in the following proposition.

Proposition 2. [CAE Equilibrium] In an equilibrium of the repeated matching market under the CAE low types always accept in stage 2 and accept in stage 1 only if $s_{j} \geq \hat{s}_{\ell}$. $\hat{s}_{\ell}$ solves equation 25 (with $x=\ell$ ), and $\underline{s} \leq \hat{s}_{\ell}<\bar{s}$. High types accept in stage 2 if and only if $s_{j} \geq \hat{s}_{2}$ (where $\hat{s}_{2}$ solves the equivalent of equation 5) and accept in stage 1 if and only if $s_{j} \geq \hat{s}_{h}$, where $\hat{s}_{h}$ solves equation 25 (with $x=h$ ). $\hat{s}_{h} \in(\underline{s}, \bar{s})$ and $\hat{s}_{h}>\hat{s}_{\ell}$.

\section{C.10 Asymmetric partial revelation environment}

We next consider an environment analogous to the ARE in the main text. In an Asymmetric Partial Revelation Environment (APRE) a male's stage 1 choice will be observed if he is present in stage 2. If we assume, conforming to intuition, that in stage one high types are at least as selective as low types, then the stage 2 reputational consequences of playing Accept will lead to a loss of continuation value similar to the intrinsic LoF. ${ }^{40}$ (However, here the loss of continuation value here may depend on one's type; we discuss this below.)

Thus we now suppose that in stage 2 a female $i$ matched to a male agent $j$ not only observes a signal $s$ but also $j$ 's stage 1 action $A_{j} \in\{A ; R\}$. Hence a male is characterized by pairs $x A \in\{\ell A ; \ell R ; h A ; h R\}$, which we will refer to as an "attribute." Denote the measure of a male with each attribute by $p_{x A}^{m}$. Then $p_{2}^{m}=p_{h A}^{m}+p_{h R}^{m}$ and as above $0<p_{2}^{m}<1$.

Suppose a female agent $i$ observes signal $s_{j}$ and past action $A_{j}$. Conditionally on being accepted and on the other observables, a female $i$ assesses the probability that her match is type

\footnotetext{
${ }^{40}$ We show the existence of this more intuitive equilibrium, but we do not rule out other equilibria. We save this for later work more focused on modelling reputation.
} 
$h$ with

$$
\operatorname{Pr}_{f}\left(h \mid s, x_{i}, A_{j}, a c c\right)=\frac{f_{h}(s) p_{h A_{j}} q_{2}^{f}\left(x_{i}, A_{j}, h\right)}{f_{h}(s) q_{2}^{f}\left(x_{i}, A_{j}, h\right) p_{h A_{j}}+f_{\ell}(s)\left(1-p_{h A_{j}}\right) q_{2}^{f}\left(a c c \mid x_{i}, A_{j}, \ell\right)},
$$

where $q_{2}^{f}\left(x_{i}, A_{j}, x_{j}\right)$ is the probability that in stage 2 a female of type $x_{i}$ is accepted by a male of type $x_{j}$ who played action $A_{j}$ in stage 1 .

A male $j$ will assess the probability that, given he is accepted and his prior action, his match is type $h$ with

$$
\operatorname{Pr}_{m}\left(h \mid s, x_{j}, A_{j}, a c c\right)=\frac{f_{h}(s) p_{2} q_{2}^{m}\left(x_{j}, A_{j}, h\right)}{f_{h}(s) p_{2} q_{2}^{m}\left(x_{j}, A_{j}, h\right)+f_{\ell}(s)\left(1-p_{2}\right) q_{2}^{m}\left(x_{j}, A_{j}, \ell\right)}
$$

where $q_{2}^{f}\left(x_{i}, A_{j}, x_{j}\right)$ is the probability that in stage 2 a male of type $x_{j}$ who played action $A_{j}$ in stage 1 is accepted by a female of type $x_{i}$.

As usual, low-type agents of both genders will always find a marriage profitable regardless of the signal they observe and the previous action of their match, since $u_{x}(\ell)>\tilde{\ell}$. Thus, for both genders, in stage 2 low types always accept. A high type agent finds accepting profitable (as before) if and only if, conditional on being accepted, the expected marriage is a favorable one. I.e., for a high-type female $i$, if

$$
\frac{p_{h A_{j}} f_{h}(s) q_{2}^{f}\left(h, A_{j}, h\right) \tilde{h}+\left(1-p_{h A_{j}}\right) f_{\ell}(s) q_{2}^{f}\left(h, A_{j}, \ell\right) \tilde{\ell}}{p_{h A_{j}} f_{h}(s) q_{2}^{f}\left(h, A_{j}, h\right)+\left(1-p_{h A_{j}}\right) f_{\ell}(s) q_{2}^{f}\left(h, A_{j}, \ell\right)}>\tilde{h},
$$

and for a high-type male $j$, if

$$
\frac{p_{2} f_{h}(s) q_{2}^{m}\left(h, A_{j}, h\right) \tilde{h}+\left(1-p_{2}\right) f_{\ell}(s) q_{2}^{m}\left(h, A_{j}, \ell\right) \tilde{\ell}}{p_{2} f_{h}(s) q_{2}^{m}\left(h, A_{j}, h\right)+\left(1-p_{2}\right) f_{\ell}(s) q_{2}^{m}\left(h, A_{j}, \ell\right)}>\tilde{h}
$$

Since the acceptance probabilities of one's match do not depend on the realization of the signal $s$ one observes, the monotone likelihood ratio property implies that the left-hand side strictly increases in the observed signal $s$ and a high-type agent uses a threshold strategy of the type "accept if and only if $s \geq \hat{s}_{2}$ " as above. Let $\hat{s}_{m}\left(h, A_{j}\right)$ denote the cutoff for a high-type male who played $A_{j}$ in stage 1 , and $\hat{s}_{f}\left(h, A_{j}\right)$ denote the cutoff for a high-type female (the low types always accept) matched to a male who played $A_{j}$.

Using the conditions above the threshold values are thus implicitly defined by

$$
\frac{p_{h A_{j}} f_{h}\left(\hat{s}_{f}\left(A_{j}\right)\right)\left(1-F_{h}\left(\hat{s}_{m}\left(A_{j}\right)\right)\right) \tilde{h}+p_{\ell A_{j}} f_{\ell}\left(\hat{s}_{f}\left(A_{j}\right)\right) \tilde{\ell}}{p_{h A_{j}} f_{h}\left(\hat{s}_{f}\left(A_{j}\right)\right)\left(1-F_{h}\left(\hat{s}_{m}\left(A_{j}\right)\right)\right)+p_{\ell A_{j}} f_{\ell}\left(\hat{s}_{f}\left(A_{j}\right)\right)}=\delta \tilde{h},
$$

for females, and by

$$
\frac{p_{2} f_{h}\left(\hat{s}_{m}\left(A_{j}\right)\right)\left(1-F_{h}\left(\hat{s}_{f}\left(A_{j}\right)\right)\right) \tilde{h}+\left(1-p_{2}\right) f_{\ell}\left(\hat{s}_{m}\left(A_{j}\right)\right) \tilde{\ell}}{p_{2} f_{h}\left(\hat{s}_{m}\left(A_{j}\right)\right)\left(1-F_{h}\left(\hat{s}_{f}\left(A_{j}\right)\right)\right)+\left(1-p_{2}\right) f_{\ell}\left(\hat{s}_{m}\left(A_{j}\right)\right)}=\delta \tilde{h}
$$

for males. This yields four different cutoff values: $\hat{s}_{f}(A)$ for a female when facing a male who accepted in stage $1, \hat{s}_{f}(R)$ for female facing a male who rejected, $\hat{s}_{m}(A)$ for a male who accepted in stage 1 and $\hat{s}_{m}(R)$ for a male who rejected.

Note that, in contrast to the CAE, we must consider cases where types are known for certain, 
conditional on previous acceptance behavior. This yields $\hat{s}_{f}\left(A_{j}\right)=\underline{s}$ if $\operatorname{Pr}\left(h \mid A_{j}\right)=1$ and $\hat{s}_{f}^{A_{j}}=\bar{s}$ if $\operatorname{Pr}\left(h \mid A_{j}\right)=0$. I.e., there is a possibility of a fully separating equilibrium where $h$ and $\ell$ play different actions in the first stage allowing perfect revelation of type in the second stage. It is, however, straightforward to bring this possibility to a contradiction with equilibrium play. ${ }^{41}$

The cutoff values defined in equations 26 and 27 satisfy

$$
\begin{aligned}
& \frac{p_{h A}}{p_{\ell A}} \frac{f_{h}\left(\hat{s}_{f}(A)\right)}{f_{\ell}\left(\hat{s}_{f}(A)\right)}\left(1-F_{h}\left(\hat{s}_{m}(A)\right)\right)=\frac{\delta \tilde{h}-\tilde{\ell}}{(1-\delta) \tilde{h}}=\frac{p_{h R}}{p_{\ell R}} \frac{f_{h}\left(\hat{s}_{f}(R)\right)}{f_{\ell}\left(\hat{s}_{f}(R)\right)}\left(1-F_{h}\left(\hat{s}_{m}(R)\right)\right) \\
& \text { and } \frac{f_{h}\left(\hat{s}_{m}(A)\right)}{f_{\ell}\left(\hat{s}_{m}(A)\right)}\left(1-F_{h}\left(\hat{s}_{f}(A)\right)\right)=\frac{\delta \tilde{h}-\tilde{\ell}}{(1-\delta) \tilde{h}}=\frac{f_{h}\left(\hat{s}_{m}(R)\right)}{f_{\ell}\left(\hat{s}_{m}(R)\right)}\left(1-F_{h}\left(\hat{s}_{f}(R)\right)\right) .
\end{aligned}
$$

The second statement in turn implies that $\hat{s}_{f}(A)>\hat{s}_{f}(R) \Leftrightarrow \hat{s}_{m}(A)>\hat{s}_{m}(R)$, i.e., if high type females are more choosy with respect to males who chose a certain action, males who chose that action will be more choosy than males who did not. Suppose that $p_{h A} / p_{\ell A}<p_{h R}\left(p_{\ell R}\right.$. Then with condition 7 from above, ensuring an interior equilibrium exists, $\hat{s}_{f}(A) \leq \hat{s}_{f}(R)$ yields a contradiction. Hence, $\hat{s}_{f}(A)>\hat{s}_{f}(R)$ if $p_{h A} / p_{\ell A}<p_{h R} / p_{\ell R}$.

To verify that this screening behavior may indeed lead to a reputational loss of face, motivating our setup above, note that expected stage 2 payoffs for the different types of male players are

$$
\begin{aligned}
& v_{2}\left(\ell A_{i}\right)=\delta \tilde{\ell}+\left(1-p_{2}\right)(1-\delta) \tilde{\ell}+p_{2}\left(1-F_{\ell}\left(\hat{s}_{f}\left(A_{i}\right)\right)\right)(\tilde{h}-\delta \tilde{\ell}) \text { and } \\
& v_{2}\left(h A_{i}\right)=\delta \tilde{h}+\left(1-p_{2}\right)\left(1-F_{\ell}\left(\hat{s}_{m}\left(A_{i}\right)\right)(\tilde{\ell}-\delta \tilde{h})+p_{2}\left(1-F_{h}\left(\hat{s}_{m}\left(A_{i}\right)\right)\left(1-F_{h}\left(\hat{s}_{f}\left(A_{i}\right)\right)(1-\delta) \tilde{h} .\right.\right.\right.
\end{aligned}
$$

This means that low males will face a reputational loss of face (i.e., $L=v_{2}(\ell A)-v_{2}(\ell R)>0$ ) if $\hat{s}_{f}(A)>\hat{s}_{f}(R)$, i.e., females are pickier when facing a male who accepted in round 1 . Note that also high type males will face a reputational loss of face (i.e., $v_{2}(h A)>v_{2}(h R)$ ) if $\hat{s}_{f}(A)>\hat{s}_{f}(R)$, using an envelope argument, noting that the effect of marginal changes of $\hat{s}_{x}($.$) will satisfy 27$.

That is, $L>0$ if $\hat{s}_{f}(A)>\hat{s}_{f}(R)$. From above we know that for any $L \leq 0$ high type males are more selective than low males, so that $p_{h A}<p_{h R}$ and $p_{\ell R}<p_{\ell A}$, which implies that indeed $\hat{s}_{f}(A)>\hat{s}_{f}(R)$. The following proposition summarizes these derivations.

Proposition 3. In an APRE in stage 2 a male player who has played accept in stage 1 has lower expected payoff than if he had played reject. That is, there is reputational loss of face in the two period matching market.

\footnotetext{
${ }^{41}$ Two possibilities emerge: $h$ 's play "always accept" and $\ell$ 's "always reject" in stage 1 , in which case an $\ell$ would have a profitable deviation by playing "always accept" in stage 1 . Second, $h$ 's play "always reject" and $\ell$ "always accept" in stage 1 , in which case an $\ell$ could do better by playing "always reject" in stage 1 , which gives players of both genders (through imitating the good signal for male, and staying in the game to access a better pool for females) a positive probability to marry an $h$ type in stage 2 , and does not decrease the probability of being accepted by an $\ell$ type.
} 